\title{
1
}

\section{The Nurse Psychotherapist and a Framework for Practice}

Kathleen Wheeler

This chapter begins with the historical context of the nurse's role as psychotherapist and the resources and challenges inherent in nursing for the development of requisite psychotherapy skills. Using a holistic paradigm, the framework for practice presented here is patient-centered and based on resilience and relationship. Mental health and illness are viewed through a cultural lens. The significant role of adverse life experiences in the development, contribution, and maintenance of mental health problems and psychiatric disorders is highlighted. A hierarchy of treatment aims is introduced on which to base interventions using a phase model for psychotherapy. This framework is based on the neurophysiology of adaptive information processing which posits that most mental health problems and symptoms of psychiatric disorders are due to a disturbance or dysregulation in the integration and connection of neural networks that occur in response to adverse life experiences. A case example is presented to illustrate the how to apply the framework proposed for psychotherapy practice.

\section{WHO DOES PSYCHOTHERAPY?}

The various disciplines licensed to conduct psychotherapy, depending on their respective state licensing boards, include psychiatrists, psychologists, social workers, marriage and family therapists, counselors, and advanced practice psychiatric nurses (APPNs) (Table 1.1). APPN is used throughout this book to include psychiatric-mental health clinical nurse specialists (PMHCNS) and psychiatric-mental health nurse practitioners (PMHNP). Educational preparation, orientation, and practice settings vary greatly among and within each discipline of practicing psychotherapists. In addition to basic educational requirements unique for that discipline, there are many postgraduate psychotherapy training programs that licensed mental health practitioners may pursue, such as psychoanalytic, family, eye movement desensitization and reprocessing (EMDR) therapy, cognitive behavioral, hypnosis, and others. Each of these training programs offer certification and require a specified amount of training for licensed mental health professionals: approximately 1 year for EMDR therapy (i.e., 40 academic didactic and 10 consultation hours for basic Parts 1 and 2 training; plus, in order to obtain certification an additional 20 consultation hours, 12 continuing educational units, 2 years' experience with a license in mental health practice, and a minimum of 50 sessions with 25 patients); and for psychoanalytic training 4 to 5 years (i.e., 4 years of coursework and supervision, ongoing practice, and one's own experience in psychoanalysis).

Postgraduate training and ongoing supervision are encouraged for APPNs who wish to gain proficiency and deepen their knowledge in a particular modality of psychotherapy. 


\section{TABLE 1.1 BASIC EDUCATION, ORIENTATION, AND SETTING OF PSYCHOTHERAPY PRACTITIONERS}

\begin{tabular}{|c|c|c|}
\hline Discipline & Education & Orientation/Setting \\
\hline Psychiatrist & $\begin{array}{l}\text { MD (medical doctor) or DO } \\
\text { (Doctor of Osteopathy); } \\
\text { 3-year psychiatric } \\
\text { residency after medical } \\
\text { school }\end{array}$ & $\begin{array}{l}\text { Biological treatment, acute } \\
\text { care, psychopharmacology } \\
\text { and specific psychotherapy } \\
\text { competencies for psychiatric } \\
\text { MD residents; often inpatient } \\
\text { orientation }\end{array}$ \\
\hline Psychologist & $\begin{array}{l}\mathrm{PhD} \text { (research doctorate } \\
\text { in psychology) or PsyD } \\
\text { (clinical doctorate } \\
\text { in psychology); } \\
\text { both usually 1-year } \\
\text { internship after } \\
\text { doctorate }\end{array}$ & $\begin{array}{l}\text { Psychotherapy and } \\
\text { psychological testing }\end{array}$ \\
\hline $\begin{array}{l}\text { Master's level } \\
\text { psychologist }\end{array}$ & $\begin{array}{l}\text { MA (Master of Arts) or } \\
\text { MS (Master of Science) } \\
\text { or MEd (Master of } \\
\text { Education) }\end{array}$ & $\begin{array}{l}\text { Psychotherapy: some modalities, } \\
\text { psychological testing }\end{array}$ \\
\hline Social worker & $\begin{array}{l}\text { MSW (Master of Social } \\
\text { Work) }\end{array}$ & $\begin{array}{l}\text { Psychotherapy: interpersonal, } \\
\text { family, group; community } \\
\text { orientation }\end{array}$ \\
\hline $\begin{array}{l}\text { Marriage and family } \\
\text { therapists }\end{array}$ & MA (Master of Arts) & $\begin{array}{l}\text { Systems and family therapy, } \\
\text { marriage counseling; } \\
\text { community outpatient } \\
\text { orientation }\end{array}$ \\
\hline Counselor & $\begin{array}{l}\text { MA (Master of Arts in } \\
\text { counseling) or MEd } \\
\text { (Master of Education in } \\
\text { counseling) }\end{array}$ & $\begin{array}{l}\text { Counseling, vocational, } \\
\text { and educational testing; } \\
\text { outpatient orientation }\end{array}$ \\
\hline $\begin{array}{l}\text { Advanced practice } \\
\text { psychiatric nurse } \\
\text { (APPN; clinical } \\
\text { specialist in } \\
\text { psychiatric nursing } \\
\text { or psychiatric- } \\
\text { mental health } \\
\text { nurse practitioner) }\end{array}$ & $\begin{array}{l}\text { MSN (Master of Science in } \\
\text { Nursing) or DNP (Doctor } \\
\text { of Nursing Practice) }\end{array}$ & $\begin{array}{l}\text { Psychopharmacology and } \\
\text { psychotherapy; group and } \\
\text { individual, sometimes family }\end{array}$ \\
\hline
\end{tabular}

Because it is highly unlikely that any one method will work for all problems for all people, the APPN who has additional skills such as hypnosis, EMDR therapy, family therapy, imagery, or ego state therapy will be more likely to help those who seek help. There are many ways to help the diverse number of patient problems and patients who seek our help, and beware of therapists who believe that "one size fits all"; in other words, if the only tool you have is a hammer, you are likely to treat every problem you encounter as a nail. 
In 2002, the American Psychiatric Review Committee mandated that all psychiatric residency programs require competency training in psychodynamic therapy (PDT), cognitive behavioral therapy (CBT), supportive and brief psychotherapies, and psychotherapy combined with psychopharmacology in order to meet accreditation standards (Plakun, Sudak, \& Goldberg, 2009). This list was further refined to what is termed the Y Model, with the stem of the $Y$ being the shared elements or common factors in psychotherapy while the arms are PDT and CBT with supportive therapy at the base of the Y (Plakun et al., 2009). Delineation of these competencies is important in that this mandate was a direct response to the increasing emphasis on medication as the treatment for psychiatric disorders and reaffirmed the importance of psychotherapy in psychiatric treatment. These core competencies in psychiatrists' education indicate a significant cultural shift that may also herald academic changes for advanced practice psychiatric nursing education.

Many factors in graduate psychiatric nursing education challenge APPNs in attaining competency in psychotherapy. One challenge for nursing education is how to teach the requisite competencies and essentials that are required in graduate nursing curricula without increasing the total credit load. To remain competitive, programs need to offer coursework that can be completed in a reasonable amount of time and with a reasonable number of credits. It is not possible in a short period-usually 2 years for most fulltime graduate master's degree nursing programs and 3 years or more for the Doctorate of Nursing Practice (DNP) degree, to attain proficiency in psychotherapy, but competency must be achieved. Psychotherapy competency was identified as necessary for all psychiatric-mental health nurse practitioner (PMHNP) programs as of 2003 (National Panel for Psychiatric Mental Health NP Competencies, 2003) and reaffirmed with the 2013 revised PMHNP Competencies (2013). With these competencies delineated and endorsed by the Commission on Collegiate Nursing Education (CCNE) for accreditation, all graduate APPN programs seeking CCNE accreditation must teach psychotherapy skills. In addition, to sit for the American Nurses Credentialing Center (ANCC) certification exam as a PMHNP, applicants are required to have clinical training in a minimum of two modalities of psychotherapy (ANCC, 2020).

Another change in nursing education that significantly impacts APPNs is the endorsement of the DNP by leaders in nursing, the NONPF, and the American Association of Colleges of Nursing (AACN). The DNP degree is envisioned as a terminal practice degree and is proposed to supplant the Master of Science in Nursing (MSN) degree for nurse practitioners by 2025 and will include a clinical research focus. Impetus for this shift came from the lack of parity with other healthcare disciplines, the high amount of credits required in current master's curricula, current and projected shortage of faculty, and the increasing complexity of the healthcare system (Dracup, Conenwett, Meleis, \& Benner, 2005). Debate continues about whether this terminal practice doctorate will enhance or dilute advanced practice. It is not clear how curricula and program requirements will evolve to provide the needed practice expertise for APPN students. Faculty need current expertise in psychiatric advanced practice to effectively teach, and concerns have been expressed about whether graduate faculty have greater academic experience than practice experience because academia traditionally rewards faculty who publish and do research. Clinical practice and teaching are often overlooked in promotion decisions, and faculty members tend to emphasize research over practice, which may not bode well for APPN faculty expertise in psychotherapy skills.

A survey of APPNs in 2009 revealed that most APPN practice time is spent prescribing, conducting diagnostic assessments, and psychotherapy with medication management but rarely solely conducting individual psychotherapy (Drew \& Delaney, 2009). A more recent national survey of 1,624 APPNs reveals that $82 \%$ provide education and $71 \%$ provide diagnostics, management, and prescribing, while $65 \%$ provide some type of psychotherapy, with $15 \%$ practicing psychotherapy alone. $65 \%$ is a significant 
increase from the 2009 data (Delaney, Drew, \& Rushton, 2019). Perhaps given the current and projected shortage of psychiatrists, the need for APPNs who can provide the full spectrum of mental health services will continue (Burke et al., 2013).

A significant challenge for graduate nursing education is the difficulty of finding preceptors and clinical sites for psychiatric graduate nursing students to practice psychotherapy. Most settings have social workers who conduct psychotherapy while the APPNs most often prescribe. This is a cost-effective approach for the agency or clinic because APPNs usually earn more per hour than social workers, but it does not provide the student nurse psychotherapist with adequate experience to practice psychotherapy. APPN students can sometimes work out an arrangement in which the student can see the preceptor's patients for psychotherapy while the psychiatric APPN preceptor manages the medication, or preceptors from other licensed mental health disciplines who are conducting psychotherapy may be asked to serve as preceptors for a limited number of hours for APPN students. In addition to the liability issues with this arrangement, space constraints, agency policy, or lack of adequate psychotherapy supervision may prohibit the student from seeing an adequate caseload of patients for psychotherapy.

A national survey of 120 academic psychiatric-mental health nursing graduate programs confirmed the scarcity of sites and found a wide range of individual psychotherapy practice hours required for students, ranging from a minimum of 50 to a maximum 440 hours in the programs for which a certain number of requisite hours are required for psychotherapy (Wheeler \& Delaney, 2005). For approximately 50\% of programs, however, no designated number of psychotherapy practice hours was required, and medication management hours were integrated along with psychotherapy. A more recent survey reveals significant differences in how APPN programs teach psychotherapy (Vanderhoef \& Delaney, 2017). The lack of consistency points to the need for more specific competencies and guidelines for what and how psychotherapy content should be taught.

Consequently, most graduate psychiatric nurses leave their graduate studies with a less than adequate knowledge base in this area, and often do not feel competent to practice psychotherapy. Faculty teaching students in graduate programs, when asked whether their students had achieved competency on graduation, felt decidedly mixed, with some stating that they did not envision a future role as psychotherapist and others suggested further training and supervision for competency to be achieved.

Working with people in the intimacy of psychotherapy is an honor, and much good can be done, as well as a great deal of harm. At vulnerable times in their lives, people see the psychotherapist as an expert, and this role often is imbued with a great deal of power and credibility. This privilege also comes with an ethical responsibility for the nurse psychotherapist to get as much training, supervision, and experience as possible in graduate studies and throughout her or his professional life. Expertise is a lifelong pursuit, and continuing education is imperative for those who wish to practice competently. Most licensed mental health professionals in other disciplines, which have considerably more psychotherapy practice in their programs than graduate psychiatric nursing programs, agree that it takes at least 10 years to become a skilled psychotherapist.

\section{Stages of Learning}

How then does one begin to learn psychotherapy? Psychotherapy is a learned skill like any other. The learning process begins with studying each component and practicing the technique and then blending it back together again with what you already know as each separate skill is acquired. Remember how you learned to take blood pressure or any other nursing skill? This can only be accomplished through learning discrete steps and practicing competencies in a skill set until that skill becomes automatic. If it seems like hard work at first, it probably means you are doing it well. 


\begin{tabular}{|l|l|}
\hline \multicolumn{2}{|c|}{ TABLE 1.2 COMPARISON OF BENNER'S MODEL AND THE STAGES OF LEARNING } \\
\hline Stages of Learning & Benner's Model \\
\hline $\begin{array}{c}\text { Unconscious } \\
\text { incompetency }\end{array}$ & $\begin{array}{l}\text { Novice } \\
\text { no experience, governed by rules and regulations }\end{array}$ \\
\hline $\begin{array}{c}\text { Conscious } \\
\text { incompetency }\end{array}$ & $\begin{array}{l}\text { Advanced beginner } \\
\text { recognizes aspects of situations and makes judgments }\end{array}$ \\
\hline $\begin{array}{c}\text { Conscious competency } \\
\text { Competency/proficiency } \\
2-5 \text { years experience, coordinates complex care and sees } \\
\text { situations as wholes, and long-term solutions }\end{array}$ \\
\hline $\begin{array}{l}\text { Unconscious } \\
\text { competency }\end{array}$ & $\begin{array}{l}\text { Expert } \\
\text { flexible, efficient, and uses intuition }\end{array}$ \\
\hline
\end{tabular}

Benner (1984) offers a model of role acquisition from novice to expert that examines the levels of competency that can be applied for the novice nurse psychotherapist. It is likely that the graduate student who is pursuing a master's degree or postmaster's certificate as an APPN has practiced as an expert in an area of specialization before graduate studies. To transition from expert back to novice is often a painful and anxiety-provoking process. The beginning nurse psychotherapist has most likely interacted professionally with many different types of patients, but there is usually much anxiety about the first session in the role as psychotherapist. There is usually no one right thing to say. In psychotherapy, there is much ambiguity and often no right answers.

Juxtaposed to Benner's model is the four stages of learning model, which may help to allay anxiety for those who are beginning to learn psychotherapy (Table 1.2). Although there is some controversy regarding who developed this model, it is thought that learning takes place in four stages:

1. Unconscious incompetence (i.e., we do not know what we do not know).

2. Conscious incompetence (i.e., we feel uncomfortable about what we do not know).

3. Conscious competence (i.e., we begin to acquire the skill and concentrate on what we are doing).

4. Unconscious competence (i.e., we blend the skills together, and they become habits, allowing use without struggling with the components).

The challenge initially for novices is that they are becoming increasingly aware of being incompetent as progress is made. This is likely to generate anxiety.

\section{Unique Qualities of Nurse Psychotherapists}

The history of the one-to-one nurse-patient relationship and nurses conducting psychotherapy is detailed by Lego (1999) and Beeber (1995). Table 1.3 highlights the important events. The late 1940s were marked by the development of eight programs for the advanced preparation of nurses who cared for psychiatric patients. An extremely important debate took place over the next few decades about the nurse's role as psychotherapist. This culminated in the 1967 American Nurses Association (ANA) Position Paper on Psychiatric Nursing, which clarified the role of the clinical specialist in psychiatric nursing as psychotherapist, and certification for the specialty began in 1979. In the 1990s PMHNP programs were developed, and this culminated in the PMHNP competencies that included psychotherapy as an essential competency required for all PMHNPs (Wheeler \& Haber, 2004). 


\section{TABLE 1.3 TIMELINE OF THE HISTORY OF THE NURSE PSYCHOTHERAPIST}

\begin{tabular}{|c|c|}
\hline 1947 & $\begin{array}{l}\text { Eight programs established for advanced preparation of nurses to care for } \\
\text { psychiatric patients }\end{array}$ \\
\hline 1952 & $\begin{array}{l}\text { Hildegard Peplau establishes the first master's in clinical nursing and a "Sullivanian" } \\
\text { framework for practice for psychotherapy with inpatients and outpatients }\end{array}$ \\
\hline 1963 & $\begin{array}{l}\text { Perspectives in Psychiatric Care first published as a forum for interprofessional } \\
\text { psychiatric articles }\end{array}$ \\
\hline 1967 & $\begin{array}{l}\text { American Nurses Association (ANA) Position Paper on Psychiatric Nursing-PCS } \\
\text { (psychiatric clinical specialist) assumes role of individual, group, family, and } \\
\text { milieu therapist }\end{array}$ \\
\hline 1979 & ANA certification of psychiatric and mental health clinical nurse specialist (PMHCNS) \\
\hline 2000 & $\begin{array}{l}\text { American Nurses Credentialing Center (ANCC) certification of psychiatric-mental } \\
\text { health nurse practitioner (PMHNP) }\end{array}$ \\
\hline 2001 & Family PMHNP ANCC Exam \\
\hline 2003 & $\begin{array}{l}\text { PMHNP Competencies developed and delineate "conducts individual, group, } \\
\text { and/or family psychotherapy" for PMHNP practice }\end{array}$ \\
\hline 2011 & $\begin{array}{l}\text { American Psychiatric Nurses Association (APNA) and International Society of } \\
\text { Psychiatric-Mental Health Nurses (ISPN) endorse PMHNP as the entry role } \\
\text { for all advanced practice psychiatric nurses }\end{array}$ \\
\hline 2013 & PMHNP Competencies revised \\
\hline 2014 & Only PMHNP Across the Life Span ANCC certification \\
\hline
\end{tabular}

The APPN role of psychotherapist has solid historical roots from the inception of advanced practice psychiatric-mental health nursing, whereas the prescribing role is a much more recent step in the evolution of the specialty. The current role of APPNs is focused on prescribing psychotropics which is more aligned with the medical model (Delaney, Drew, \& Rushton, 2019).

After the issue of whether nurses should do psychotherapy was resolved, the literature examined the unique qualities that nurses might possess as psychotherapists compared with those in other disciplines who practice psychotherapy. Several strengths were cited: nurses have the ability to be patient because they have worked with the chronically ill and have respect for others' limitations; nurses are realistic and possess excellent observational skills, resourcefulness, innovation, and creativity (Smoyak, 1990); nurses are able to view the patient in a holistic way, understand crisis orientation, and have a knowledge of general health concerns (Lego, 1992); and nurses are familiar with the daily life and experience of the hospitalized patients (Balsam \& Balsam, 1974). Nurses usually have had a breadth of life experience and exposure to many different ages, ethnicities, occupations, socioeconomic status, cultures, and personalities. The novice nurse psychotherapist is well served through experience with communicating and connecting with those from diverse backgrounds. Nurses being close to the patient's everyday experience is crucial for connection and collaboration. This connection is reflected in the public perception of nurses as positive and trustworthy. In 2019 for the 18th year in a row, the Gallup poll found that nurses top the list of most ethical professions, with Americans rating nurses among the most trusted professionals. 
Eighty-five percent of respondents rated nurses' honesty and ethics as "very high" or "high" with medical doctors rated third at 65\% (Gallup, 2020).

An additional quality that nurses bring to the role of psychotherapist is a pragmatic, problem-solving approach using the nursing process as an overall framework for practice. Usually, the patient has tried many things to feel better, and therapy is often a last resort. The patient's problems have brought the person into treatment, and if these problems could be solved outside of therapy with friends or family, he or she would have already done so. The problem-solving approach needed in the psychotherapeutic process is the same as in the nursing process. Both involve an assessment, diagnosis, plan, intervention, and evaluation. Nurses are used to collaborating with patients and thinking about what will solve the problem, what the patient's perspective is, what the person wants, and what the patient's strengths are. These approaches are derived from a problem-solving, health-oriented, holistic model fundamental to nursing practice and the nurse-patient relationship.

In my experience working with graduate psychiatric nursing students, this problemsolving approach is useful but one that novice nurse psychotherapists often struggle with. Because nurses are used to taking care of people and are action oriented, beginning students often want to rescue the patient and help the patient to feel better. Helping the patient feel better is not the main goal of psychotherapy, and a focus on amelioration of symptoms may even be counterproductive to the process, although feeling better overall most likely will be a by-product of successful therapy. In a well-intended effort to help the person feel better, the nurse may be too directive and offer fix it suggestions, and this is antithetical to promoting empowerment. Letting the psychotherapeutic process unfold takes time, and that has typically not been a part of nursing practice, especially within the current healthcare system.

\section{Requisites for Nurse Psychotherapists}

Nurse psychotherapists have the honor of participating in the healing process, and as nurse theorists Dossey and Keegan (2013) point out, in the nurse-patient relationship, the nurse enters into a shared experience or field of consciousness that promotes the healing potential of others. Through consciousness, intent, and presence, the nurse psychotherapist's therapeutic use of self facilitates others in their healing. To counter the learned patterns of nursing practice (i.e., busyness, task focused, and control), the nurse psychotherapist needs to cultivate reflection, mindfulness, and patience. According to Dossey and Keegan (2013), qualities essential for nurse healers include expansion of consciousness and continuing one's own journey toward wholeness and resilience. This can be accomplished through many different venues: nature, relationships, your own therapy, ongoing supervision, meditation, mindfulness, self-awareness exercises, spiritual practices, chanting, prayer, journaling, openness to receiving one's own healing treatments, and reflective activities such as hiking, walking, and yoga. Research has shown that the regular practice of mindfulness improves empathy, insight, immune function, attention, and emotional regulation (Dahlgaard et al., 2019). These changes correspond to changes in the brain that include increased activity and growth of regulatory and integrative regions which foster resilience.

Mindfulness is a skill that can be learned through practice and discipline and used as a tool in the psychotherapeutic process. The vast literature on the development of mindfulness crosses many disciplines and orientations, from Buddhism to psychoanalysis. Mindfulness is discussed more fully in Chapters 17 and 18. Safran and Muran (2000) state that mindfulness in psychotherapy has three characteristics:

(a) The direction of attention, (b) remembering, and (c) nonjudgmental awareness. The initial direction of attention involves intentionally paying attention to and observing 
one's inner experience or actions. This involves cultivating an attitude of intense curiosity about one's experience. In mindful meditation, the individual can initially cultivate the ability to attend by focusing the attention on an object (e.g., the breath) and then noting whenever his or her attention has wandered and returning it to the intended focus of attention. By noting whatever one's attention has wandered toward (e.g., a particular thought or feeling) before redirecting one's attention, the individual develops the ability to observe and investigate his or her experience from a detached perspective rather from being fully immersed in or identified with it. (p. 59)

Peplau (1991) stressed the need for self-awareness in the nurse-patient relationship and stated: "The extent to which each nurse understands her own functioning will determine the extent to which she can come to understand the situation confronting the patient and the way he sees it" (p. x). However, with the rise of psychopharmacology and biological psychiatry, self-awareness has not been a priority. Self-awareness is key to understanding others, and it reduces the likelihood that therapists will act out their own agendas and use patients for gratification or self-esteem needs. For example, one novice nurse psychotherapist was so rewarded emotionally by his work with a particular patient that he went out of his way to meet with her when she needed him and to schedule additional office hours when he would not normally be in the office. The patient responded with gratitude, which enhanced the self-esteem of the nurse who was conscientious and overly responsible for this patient. It was only through supervision that he began to understand how his need for recognition fueled the overly accommodating stance; how his objectivity about the psychotherapeutic process had been compromised; and how this cultivated an unhealthy dependency in the patient.

Peplau (1991) says that there is a tendency for all those doing therapeutic work to generate inferences from limited data and to assume that these data are complete. It is only natural that we would try to fit the problem into our own limited schemata of experiences, but the richness of clinical data belies this belief. Attributing motivation to one simple reason, such as "she's borderline and manipulative," is simplistic and may assuage our anxiety but does not account for complex, multifaceted interactions and contributions that are more often the norm than the exception. Symptoms are usually multidetermined and have many different contributing factors.

Overdeterminism refers to the idea that a problem most often has many different causes. The patient may not be able to provide a full description of these contributions and most likely is unaware of the multiple reasons for the current symptom. For example, a young woman with bulimia may have factors that contributed to the development of her problem: a history of sexual abuse, feelings of deprivation and neglect in her family, a recent loss in her family, a genetic predisposition, a fear of weight gain, cultural pressures about weight, an overemphasis on weight in her family, an inability to self-soothe, a hormonal imbalance, the stress of a new job, and a best friend who is also bulimic. The friend with the same problem may have a few of these contributing factors and others, such as conflict in her home with an abusive, alcoholic father; a depressed, unavailable mother; and financial difficulties that contribute to the instability of her home environment and compromise her ability to manage her emotions. There are no simple answers, and two people with the same problem may have developed and maintained their symptoms for different reasons. Many factors, such as genetics, prenatal insults, parent-child interactions, abuse, neglect, school and social environments, family dynamics, and physical illness, have been studied, and all have been found to play a role in the cause of psychiatric disorders and mental health problems.

We all have preconceptions that are brought to every situation. It is not as important to eliminate these as to be aware of what they are and how they may influence our work. The extent of a nurse's self-knowledge determines the extent to which he or she can 
understand another person. Neuroimaging studies have confirmed that being aware of another's mind is related to a person's ability to monitor his or her own mental state (Siegel, 2012). A person does not have to be a paragon of mental health to help another. Some feel that to be truly empathic, a person should have experienced psychological suffering, which can serve to deepen the work in psychotherapy. Most expert therapists consider personal therapy and supervision essential for the novice psychotherapist to cultivate emotional genuineness, authenticity, and objectivity. Supervision is not therapy, but it does assist the therapist in discussing difficult cases and understanding his or her own blind spots and how personal issues may impact the therapeutic relationship. Ongoing group or individual supervision after graduation is necessary for continued growth and an ethical practice. Expert psychotherapists usually seek supervision and consultation throughout their professional lives. A sample of suggestions for presenting a case that may be covered in supervision is included in Appendix 1.1.

Irvin Yalom cogently makes a case for therapy for the therapist:

Therapists must be familiar with their own dark side and be able to empathize with all human wishes and impulses. A personal therapy experience permits the student therapist to experience many aspects of the therapeutic process from the patient's seat: the tendency to idealize the therapist, the yearning for dependency, the gratitude toward a caring and attentive listener, and the power granted to the therapist. Young therapists must work through their own neurotic issues, they must learn to accept feedback, discover their own blind spots, and see themselves as others see them; they must appreciate their impact upon others and learn how to provide accurate feedback. (Yalom \& Ferguson, 2002, pp. 40-41)

The student of psychotherapy who undergoes his or her own psychotherapy has a model for what the psychotherapeutic process is and understands the power and the process of psychotherapy in an immediate, experiential way that no amount of reading or didactic study can convey. Many expert psychotherapists report that they have experienced various modes of psychotherapy and this has enhanced their own technique as the skills others use are incorporated into their own practice.

In addition to self-awareness, enhancing one's own resilience through promoting self-care is fundamental in caring for others. Self-care strategies start with exercising regularly, eating healthily, spending time with friends, getting enough sleep, and limiting work hours to avoid exhaustion. When a flight takes off, the airline attendant announces that all adults must put the oxygen mask over their faces first before securing the mask on a child. This is an appropriate metaphor for all caregivers. Much has been written about the trauma inherent in nursing. Various terms have been used to describe this phenomenon, such as burnout, compassion fatigue, and vicarious or secondary traumatization. A 2017 survey of employed RNs in hospitals reported that $63 \%$ experience burnout (Kronos, 2017). Burnout may occur for many reasons: our collective history as a profession of women, the patriarchal medical system and nurses' subservient role, stressful healthcare environments, and caring for and witnessing trauma and pain in others. Most often, personal and professional trauma is unrecognized and therefore unaddressed. Sequelae of exposure to other's trauma may include fatigue, depression, anger, apathy, detachment, headaches, insomnia, and gastrointestinal distress (Foli \& Thompson, 2019), all of which mitigate against the ability to adopt the psychotherapeutic stance necessary for conducting psychotherapy. It is only in the recognition and processing of one's own trauma that one can transcend it and be of help to others. A resource for understanding and overcoming compassion fatigue can be found at www. edumed.org/resources/compassion-fatigue-online-guide.

Competencies for Trauma and Resilience for Nursing Education have been developed and include self-resilience competencies for APPNs (Wheeler \& Phillips, 2019). 
These competencies build on those for undergraduate and graduate level education and are copyrighted. The full document with subcompetencies are included online in the faculty resource website and at www.acesconnection.com/blog/ trauma-and-resilience-competencies-for-nursing-education.

\section{HOLISTIC PARADIGM OF HEALING}

In contrast to the biomedical model's goal to cure with symptom relief treatment, the goal in a holistic paradigm is healing (see Figure 1.1). This is an important distinction, because curing is not always possible but healing is (Dossey \& Keegan, 2013). The word heal comes from an old Anglo-Saxon word haelen, which means "to become whole, body, mind, and spirit within oneself"; but it can also be defined in a broader context as being in "right relationship" with oneself, others, and our world. Mariano defines healing as "an emergent process ... bringing together aspects of one's self and the body, mind, emotion, spirit, and environment at deeper levels of inner knowing, leading to an integration and balance" (2013, p. 60). Each component is interdependent and interrelated, based on the premise that when there is a change in one part of the system, the change reverberates in all dimensions. For example, minor changes in one's emotions may potentiate a change in all other spheres as well as in the person's relationship with others and his or her world. Conversely, a change in the context or relationships with others may create changes in other dimensions (e.g., body, mind, emotion, spirit) of the person. The context or background is the person's culture as mediated by the person's family and relationships.

Some of the goals of psychotherapy include the reduction of symptoms, improvement of functioning, relapse prevention, increased empowerment, and achievement of the specific collaborative goals set with the patient. Within the biomedical model, symptoms are often thought to be the cause of the patient's problem and psychotropic medications are prescribed to target specific symptoms in an effort to eliminate or reduce the symptoms. For example, prescribing a selective serotonin reuptake inhibitor (SSRI) to increase serotonin levels is thought to treat the underlying cause of the depressive disorder. However, whether this chemical imbalance causes depression or coexists with some depressive disorders is a matter of speculation.

In contrast, in a holistic model, symptoms are seen as a form of communication and are useful for understanding the meaning of the dysregulation and disharmony that are
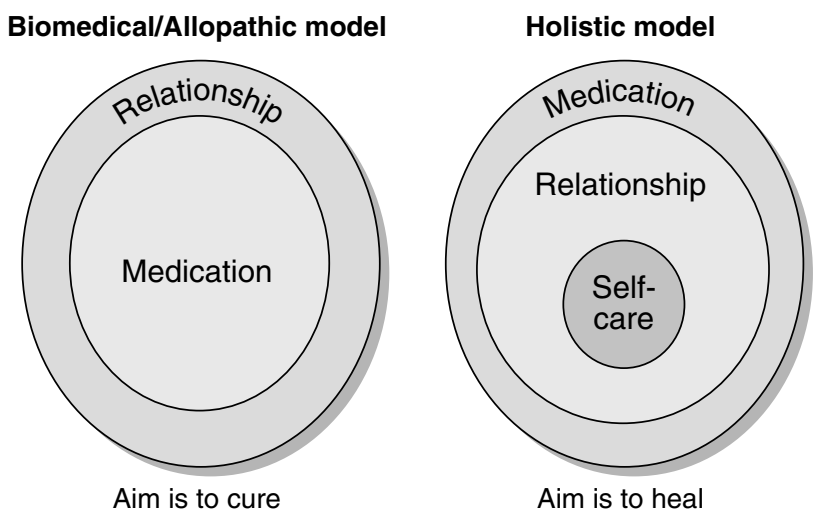

FIGURE 1.1 Paradigms of care. 
occurring for this person at a given time. By eliminating the symptoms with medication, we are essentially "shooting the messenger." Often therapists find that therapy works best with full access to emotion; that is, if the person's emotions are damped down by benzodiazepines or other psychotropic medication, psychotherapeutic work may be compromised. For example, CBT seeks to allow the patient to become more comfortable with sensations and concomitant emotions related to panic attacks so that automatic thoughts about how dangerous these feelings seem can be confronted. If the patient reaches for medication for quick relief, the person may lose motivation to continue the treatment (Cloos \& Ferreira, 2009). Of course when the patient's functioning is impaired, psychotropic medications do have their place in treatment. However, reframing symptoms as communication changes the way we view the relation of the problem to the person and enhances our ability to hear the meaning of the symptoms as we listen to our patient as well as access the person's emotion in order to facilitate the treatment of the patient.

The holistic paradigm is consistent with the mandate for recovery-oriented behavioral care. The Substance Abuse and Mental Health Services Administration (SAMHSA, 2020) provides a definition of recovery: "A process of change through which individuals improve their health and wellness, live a self-directed life and strive to reach their full potential." In addition to holistic care, elements of care aimed toward recovery include hope, respect, multidimensional care through many pathways, person-driven, supported by peers, culturally based, addresses trauma, supported through relationships and social networks, involving individual, family, and community strengths and responsibilities. Research has identified five gold standards of recovery for patients: hope, self-esteem, empowerment, self-responsibility, and a meaningful role in life (Livingston \& Boyd, 2010; Siu et al., 2012). Practitioners who are recovery-oriented recognize the strengths and power of each person within the context of his or her life. The vehicle for recovery is through partnership and relationship with the practitioner and others so that the person is the driver of his or her own healing process (SAMHSA, 2020).

These elements and gold standards for recovery may feel familiar to APPNs because nurses have been educated to look at the context of the patient's life as they work in the reductionistic, symptom-oriented environment of the psychiatric biomedical paradigm. Biomedical psychiatry is based on a descriptive/biological approach of specialized knowledge that treats individuals as members of a diagnostic group. The diagnosis is based on observable clusters of symptoms or behaviors and there is no assumption about causation except for the Diagnostic and Statistical Manual of Mental Disorders (5th ed.; DSM-5; American Psychiatric Association [APA], 2013) category of trauma-related disorders. What is considered pathological is determined by societal values and behaviors that are considered acceptable at the time by a panel of, until most recently, exclusively psychiatrists. For example, the DSM-III considered homosexuality a psychiatric disorder while the DSM-5 includes new diagnoses such as Binge Eating and Hoarding Disorders (APA, 2013).

The diagnosis may not tell us very much about the person sitting in front of us. The nurse is often the only person caring for the patient who sees the whole picture. The nurse knows the patient as "a grandmother who lives alone in a walk-up, estranged from her daughter and often terrorized by her own internal demons" while those practicing from a medical model might describe the same person as "an 88-year-old elderly woman with bipolar disorder." The former is relevant about who the person is while the latter tells us nothing about the uniqueness of that individual. Indeed the nurse practicing from a holistic paradigm respects the complexity of the person, and historically, this has been the foundation for nursing practice. 


\section{Relationship}

Relationship has been considered foundational for psychiatric nursing since Peplau's seminal work in 1952 (D'Antonio, Beeber, Sills, \& Naegle, 2014). The healing that takes place in psychotherapy occurs through the relationship between the therapist and the patient. Lego (1992) maintained that psychiatric nurses develop "a relationship designed to change the patient's interpersonal situation, changing the intrapsychic situation, thus changing the brain chemistry" (p. 148). Forchuk and associates (1998) observed that the nurse-patient relationship is the "active ingredient" in therapeutic change. Raingruber (2003) concurs and says that relationship and nurturing are hallmarks of psychiatric nursing. Dossey and Keegan (2013) say that the healing relationship occurs through the expansion of consciousness, during which a sacred space is created.

Emotional connection promotes interpersonal attunement, attachment, and coregulation of physiological states (Schore, 2019). Emotional connection with the patient through relationship has been found to be important for successful psychotherapy outcomes with $50 \%$ of positive outcomes due to therapist, relationship, and expectancy while the specific technique or theory used by the therapist accounts for only $17 \%$ of improvement; $33 \%$ of the variance is due to extratherapeutic factors such as spontaneous remission, patient, and community factors (Norcross \& Lambert, 2019). The ability of the patient to connect through collaboration depends on the therapist's skills and on the patient's emotional developmental level, with some patients much better able to join in collaboration than others. Tryon and Winograd (2002) found that the more troubled, resistant, less-motivated patients are those most likely to need help and the least likely to engage and collaborate with therapists. Chronically disempowered patients, especially those who have been severely traumatized in childhood, often are unable to connect with others and use support to reach new solutions. The challenge for the APPN is how to facilitate connection, particularly with patients who have difficulty with relationships. Inherent in this connection is the APPN's curiosity and openness to learning about another's experience. The receptivity and openness of the therapist to what is presented offers the patient a model for developing curiosity about him- or herself and for an observing ego. It is thought that this capacity to develop the ability to observe one's own behavior with nonjudgmental curiosity is a hallmark of emotional health.

Caring in the nurse-patient relationship has been identified as essential for practice (Dossey \& Keegan, 2013; Morse, Solberg, Neander, Bottorff, \& Johnson, 1990; Schoenhofer, 2002; Watson, 2012). Caring encompasses and expands Carl Rogers's idea of unconditional positive regard that has been adopted by most mental health disciplines as essential to helping relationships (Rogers, 1951). A phenomenological study delineated the characteristics of the APRN-patient relationship that are foundational to caring (Thomas, Finch, Schoenhofer, \& Green, 2004). They include the mutuality of nonromantic love based on a genuine knowing of the person, trust, and respect reflected in an acceptance of and authentic appreciation for the other. Every person is approached with acceptance with the nurse and patient as coparticipants in the process of healing. Inherent in caring is respect for the autonomy and agency of the other person. Fundamental to caring is the understanding of another person's unique configuration of attitudes, feelings, and values from that person's perspective.

The nurse psychotherapist creates a healing presence of acceptance, patience, lovingness, nonjudgmental attitude, understanding, good listening skills, honesty, and empathy. These qualities are the essence of presence (McKivergin, 1997) and allow the nurse psychotherapist to "be with" rather than "doing to" the patient. Bunkers (2009) says, 
"True presence involves listening to what is important to the other and listening to what the meaning of a situation is in the moment for that person" (p. 22). Scaer (2005), a neurologist specializing in trauma, says that presence involves a personal interaction that contributes to physiological changes in the person. He states, "This healing, empathic presence affects and alters the parts of the brain that process pain, fear, anxiety, and distress" (p. 167). Presence may facilitate healing through mediation of neurotransmitters and hormones that promote optimal autonomic functioning.

The antithesis to empowerment is authority; in this situation, the therapist knows what is best for the person. The process of psychotherapy cultivates dependency because there is unavoidable inequality in the relationship with the patient, who naturally feels disempowered by needing help at a vulnerable time. This reality and the inevitable transference-countertransference responses create dependent feelings in the patient. The psychotherapist's competence lies in understanding that the patient's autonomy is always in the foreground of the process. The overall goal for patients is to deepen their understanding of themselves in order for them to make their own decisions. Caring is fundamental to creating an atmosphere conducive to the cultivation of relationship and empowerment.

\section{Resilience}

Both relationship and resilience are overarching pantheoretical concepts that apply to all approaches of psychotherapy and practice settings. The term resilience refers to the ability of an individual, family, or community to cope with adversity and trauma, and adapt to challenges through individual physical, emotional, and spiritual attributes and access to cultural and social resources (adapted from SAMHSA, 2014). In fact, there is speculation that surviving a crisis can actually be a growthpromoting experience for some people. However, other research supports that resilience and posttraumatic growth are inversely related; that is, those who cope well and are resilient after a traumatic event retain equilibrium and do not need to find positive meaning to the event while those who emerge with posttraumatic growth feel the need to reframe the event as positive (Levine, Lalufer, Stein, HamamaRaz, \& Solomon, 2009). A more recent study supports that moderate resilience and emotional intelligence is associated with the most growth ( $\mathrm{Li}, \mathrm{Cao}, \mathrm{Cao}, \& \mathrm{Liu}$, 2015). That is, there is a curvilinear relationship between posttraumatic growth and resilience.

An elegant yet simple resilience model based on neurophysiology is proposed by Elaine Miller-Karas and deepens our understanding how to help patients access their resources (2015). Miller-Karas says that one's resilient zone (RZ) is an internal state of adaptabiliy and flexibility that is regulated by our nervous system. We feel at our best and can think clearly and deal effectively with life when we are in our RZ. She states that there is a natural biological rhythm of the autonomic nervous system between the sympathetic nervous system and the parasympathetic nervous system and this corresponds to the RZ. The RZ is also referred to as the window of tolerance (Ogden, Minton, \& Pain, 2006), or the therapeutic window of arousal (Siegel, 2012). Porges's polyvagal theory refers to the state of our nervous system as the myelinated ventral vagus social engagement response (Porges, 2011). The brain and the body communicate with each other unconsciously through the vagus nerve. Chapter 2 discusses the polyvagal theory and the neurophysiology of the RZ and its role in well-being and healing trauma. The skilled psychotherapist helps the person through various techniques that assist the person to mediate the autonomic nervous system and stay in their RZ, that is, not too hyperaroused (sympathetic system) and not too hypoaroused (parasympathetic 
system; see Figure 1.2). This is the optimal physiological state for the work of therapy and reflects the person's natural rhythm and flow of energy and vitality. Although the person may feel sad, happy, angry, and other emotions when in their RZ, the person is able to both feel and think at the same time.

If the person becomes too anxious and hyperaroused, resistances or defenses may increase, and the work of therapy will be thwarted, perhaps not consciously, but nevertheless, the person's brain will not be able to integrate memories or gain insight. Immediate strategies in a session to decrease arousal levels might include deep breathing exercises or imagery. There are also many patients who have suffered significant trauma and are in a chronic state of either hyperarousal or hypoarousal or swing from one physiological state to the other (see Figure 1.3). If the person is chronically hypoaroused, he or she may be unable to access emotions. Strategies for hypoarousal to increase arousal might include focusing on sensations in the body, mindfulness exercises, and self-regulation strategies. Self-regulation refers to one's ability to manage emotions and behavior and is further discussed in Chapter 2. Psychotherapy helps those with emotional dysregulation to widen and strengthen their RZ.

Severe trauma has been found to override constitutional, environmental, genetic, or psychological resilience factors (De Bellis, 2001). Studies have shown that factors that enhance resilience include the presence of supportive relationships and attachments as well as the avoidance of frequent and prolonged stress (Herrman et al., 2011). These factors are not inborn but can be fostered through psychotherapeutic interventions that focus on the strengths of the person, reducing risks, and improving relationships. Chapter 11 further discusses the RZ specific strategies to enhance resilience. Relationships form the foundation of resilience and serve to create new experiences that promote neuronal and synaptic connections that allow for learning new meaning for prior adverse experiences.

Figures 1.2 and 1.3 illustrate a user-friendly model that the APPN can teach patients at the beginning of therapy when making a treatment plan. Illustrating the RZ on paper and then drawing Figure 1.3 helps patients to understand their symptoms without judgment as something physiological that is happening to them. Many patients have no understanding why they feel so out of control, anxious, depressed, or numb and providing a simple explanation based on neurophysiology with visuals such as Figures 1.2 and 1.3 can be very helpful and destigmatizing. The message conveyed is, "It is not what is wrong with you but what has happened to you,"

One's Resilient Zone is the best physiological state for thinking clearly and functioning well.

Sympathetic Nervous System

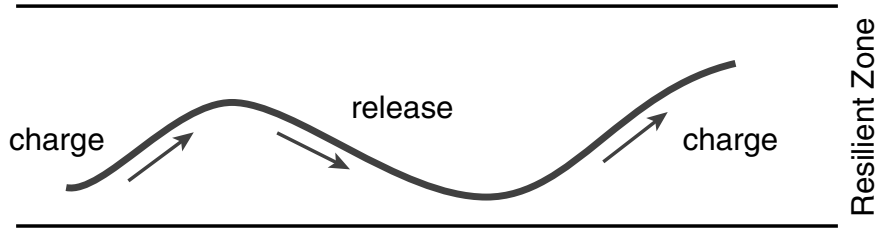

Parasympathetic Nervous System

FIGURE 1.2 Therapeutic window of arousal/tolerance, or resilient zone. 


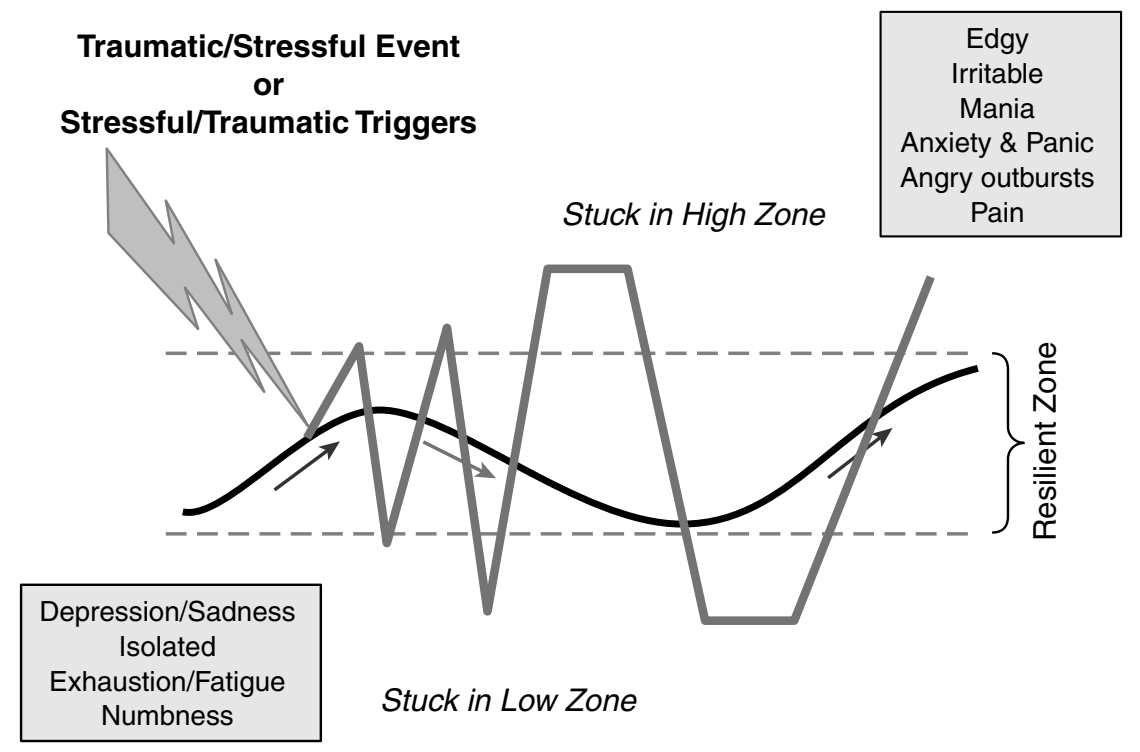

FIGURE 1.3 Out of the resilient zone.

Source: Adapted by Elaine Miller-Karas from an original graphic by Levine/Heller. Reprinted with permission from the Trauma Resource Institute, Claremont, CA.

which lays the foundation for a trauma-informed approach to your work together. Techniques and resources to widen or strengthen the patient's RZ will be further discussed within the context of the various psychotherapy approaches presented in this book. See Chapters 2 and 11, for a fuller discussion of the underlying neurophysiology of the RZ.

\section{ANXIETY}

Understanding, assessing, and managing anxiety is a cornerstone of Peplau's Interpersonal Relations Model for Nursing (1991). Anxiety is ubiquitous in the psychotherapeutic process, and the skilled APPN understands how to assist the patient in managing anxiety. Anxiety creates feelings of helplessness, which disempower the patient and prevent healing. Wachtel (2011) says:

One of the chief aims of the psychotherapist is to help the patient overcome the fears and inhibitions that have led him to react to his normal and healthy feelings as if they were a threat; to help him reappropriate parts of himself that have been dissociated from full awareness, that have motivated avoidances, and that are likely to generate still further areas of vulnerability, deficits in crucial skills in living, and impediments to the very relationships that could in principle be correctives to the debilitating anxiety. (p. 87)

For the most part, people seek psychotherapy because anxiety or the effects of anxiety have in one way or another interfered with functioning. Sometimes, a person is seeking help for the anxiety itself, such as in cases of panic attacks or phobias, but often the presenting issue is related to the results of the person's efforts to avoid anxiety. For example, a person with borderline personality traits may present with depression as 


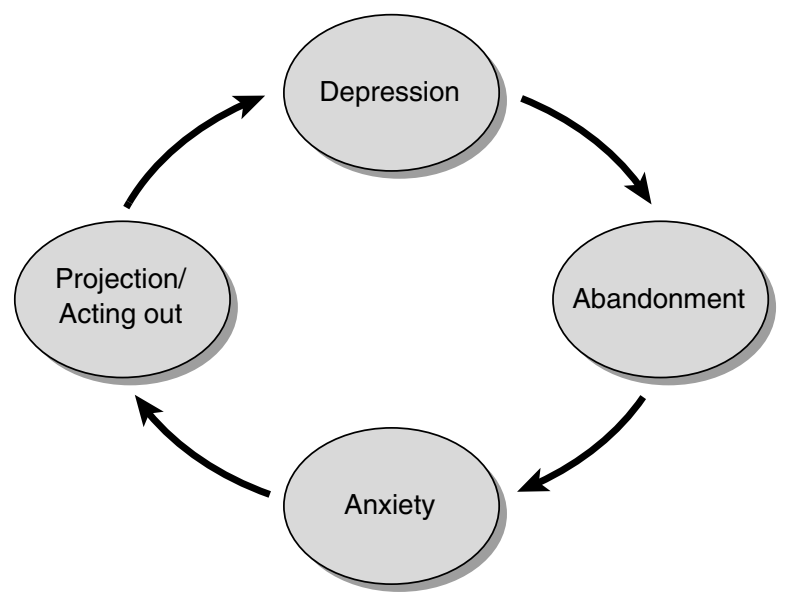

FIGURE 1.4 Cyclical psychodynamics of a person with borderline personality disorder.

a result of a lost relationship, but the central issue is a vulnerability to abandonment anxiety. It is likely that in the person's zeal to avoid the feared abandonments, that person inadvertently creates the very situation that he or she is trying so hard to avoid (Figure 1.4). Wachtel (2011) calls this cyclical psychodynamics, which is explained further in Chapter 5.

Inherent in all the theoretical approaches and basic principles discussed in this textbook is the centrality of anxiety as key to the patient's problems and the management of anxiety as key to solving these problems. In the safety of the therapeutic relationship, patients are encouraged to tolerate the feared experiences, memories, and thoughts. Cozolino (2017) says that a major role for the therapist is to assist the patient in using anxiety as a compass to explore unconscious fears. In deepening his or her understanding of anxiety as a trigger for avoidance or acting out, the person can then approach with curiosity what is feared. Strategies for working with anxiety are central to all therapy approaches. For example, behavioral techniques such as desensitization or flooding may be taught and increase anxiety initially, with the hope of decreasing anxiety later, so the person can face what was fearfully avoided. Cognitive techniques may involve "restructuring" thinking so that the threat that is anxiety-provoking is not considered as dire as originally believed. Psychodynamic techniques use interpretations to deepen the person's understanding of anxiously avoided thoughts, wishes, and feelings by making the unconscious conscious in order to understand the cause of anxiety.

For those patients with chronic hyperarousal and anxiety disorders, their RZ may be too narrow, and strategies and resources that help to widen their RZ may be needed. These can include basic stress management activities, such as exercise, decreasing caffeine intake, relaxation exercises, and imagery. A useful weekly plan for increasing resources and a weekly goal sheet is included in Appendices 1.2 and 1.3. Asking the person what he or she does to relieve anxiety or stress is part of good history taking, and developing a plan together that is not overwhelming is essential. Books such as Bourne's The Anxiety and Phobia Workbook (2015) can be very helpful and an important adjunct to therapy. The patient can be asked to read a chapter and complete the exercises in selected relevant chapters, and the next session is begun with a discussion about the person's experience with the material. Additional strategies to manage anxiety are especially important for those with dissociation and trauma-related disorders, and these are discussed further in Chapter 17. 
However, a caveat is in order. Workbook exercises are only an adjunct to treatment and do not take the place of the real work in therapy, which is co-constructing a narrative and connecting through a therapeutic relationship. A consistent finding is that treatment manuals do not correlate positively with treatment outcome (Moncher \& Printz, 1991; Strupp \& Anderson, 1997; Truijens, Zühlke van Hulzen, \& Vanheule, 2019). This may in part result from the constraints on creativity and flexibility with such a "cookbook" approach that is not context driven. Often, novice psychotherapists feel more comfortable with these structured approaches and with "doing" things; thus, it may help to manage the therapist's anxiety more than it does the patient's. In addition to monitoring the patient's anxiety, the beginning APPN must be aware of and manage his or her own anxiety.

It is easy to see why therapy in and of itself is highly anxiety provoking. Change, even a positive change such as we hope occurs in psychotherapy, is anxiety provoking. A seminal study by the Menninger Foundation found that patients who had positive outcomes from psychotherapy often reported an increase in anxiety, but they had learned to use anxiety as a signal rather than as a reality that danger was present (Siegel \& Rosen, 1962). In the safety of the therapeutic relationship, the person is exposed to what has been avoided; and as the person begins to change toward healthier ways of functioning, increased anxiety is inevitable. It is important for the therapist to keep this in mind and monitor the patient's anxiety level as the therapeutic process unfolds. If anxiety becomes too unbearable in psychotherapy, there may be acting-out behaviors and increased resistance to change, or the person may leave treatment prematurely.

Anxiety is inherent in any new enterprise, and learning psychotherapy can be particularly anxiety provoking. In psychotherapy, we are trying to make sense of what is going on, and new information is emerging in every minute in our interaction with patients. One way the brain deals with ambiguous situations is to categorize information. This is largely what diagnosing is about-categorizing and labeling patients through a list of behavioral characteristics. The brain tries to fit the person into what is familiar, and this limits our ability to approach the patient with openness and without preconceptions. As anxiety increases, our focus becomes more limited, and it is harder to maintain the openness required to achieve a nonjudgmental, observational stance. Developing selfawareness about one's own anxiety is essential in empowering the therapist to allow the space needed for the relationship to develop.

\section{MENTAL HEALTH AND CULTURE}

To practice psychotherapy, the therapist must have a model on which to base interventions and some idea of what constitutes a mentally healthy person. Freud's simple idea that the goal of therapy is to be able to work and love remains relevant, because it can be applied generally to all cultures and people. In contrast, Sullivan (1947) thought that self-awareness was key to mental health and said, "One achieves mental health to the extent that one becomes aware of one's interpersonal relations" (p. 207). A more contemporary idea is offered by Siegel (2012) and is based on a systems perspective. He says that mental health emerges from integration in the brain/body through relationships. Integration is the core of resilience and vitality and reflects coherence of one's own states of mind. "Internal integration allows for vital interpersonal connections that are themselves integrative." (p. 351) Integration is accomplished through information processing that links disparate parts into a functional whole. The neurophysiological underpinnings of integration are explained further in Chapter 2. 
BOX 1.1 Qualities of Self-Actualization
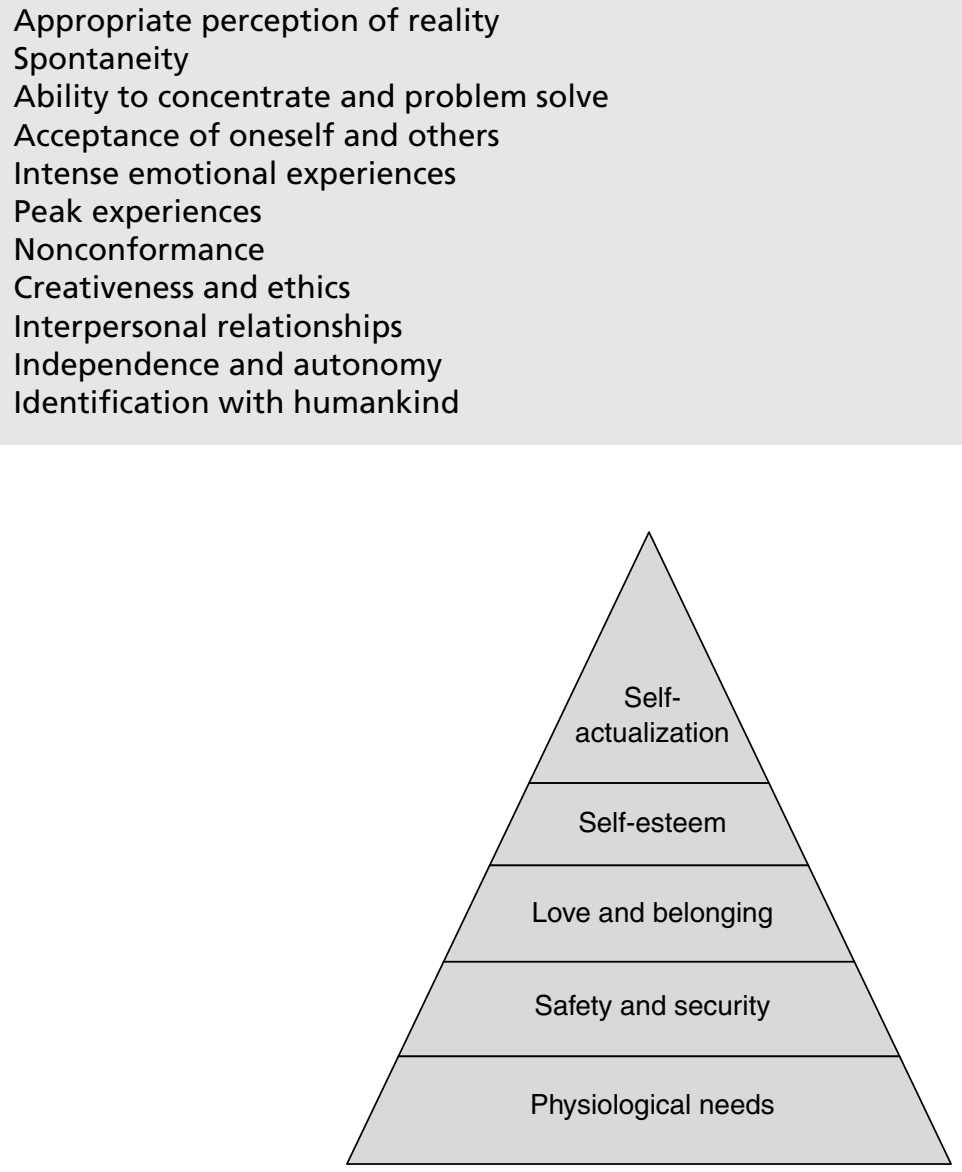

FIGURE 1.5 Maslow's hierarchy of needs.

Source: Adapted from Maslow, A. H. (1972). The farther reaches of human nature. New York, NY: Viking.

Maslow delineated the ideal of a mentally healthy person as one who is self-actualized and who has the characteristics summarized in Box 1.1. Maslow's hierarchy of needs framework for problem solving is useful in conceptualizing the priority of patient needs (Maslow, 1972). Lower-level needs must be met before higher-level needs can be addressed. Meeting physiological needs is essential, with physical and emotional safety and security next (Figure 1.5). Safety in the world and the therapeutic relationship is essential to enable disclosure so that higher-level needs on the continuum, such as love, self-esteem, and self-actualization, can be achieved. This model is not fixed in that an individual may achieve self-actualization and then be faced with a trauma and have a need for physiological safety that would then take priority over self-actualization and needs higher in the hierarchy.

It is apparent from reviewing the characteristics of self-actualization in Box 1.1 that the meaning of mental health is culture bound; Maslow's self-actualized person, embodying independence, autonomy, individuation, and nonconformance, is largely a Western idea. For example, Eastern cultural values of interdependence, communal integration, and group harmony which does not fit with Western ideas of self-actualization. Some 
dimensions of this framework may apply to certain cultures but not to others. Cultural relativity is a term that Horowitz (1982) identified as important to consider in any discussion of mental health; behavior that is considered normal or abnormal depends on social and cultural norms.

Culture is an integral part of all relationships. Our cultural context shapes our perceptions, emotions, attributions, judgments, and ideas about ourselves and others (Barrett, 2017). The powerful influence of culture permeates all dimensions of our life in a way that is often unconscious. We are all multicultural in the sense that we belong to many different cultures simultaneously. For example, a young man who recently returned from combat belongs to the military culture, which values winning in battle and requires following orders and acting bravely. He may return to a society that does not value the war he fought and find a clash of values on his return. He may also belong to an Irish cultural heritage that does not sanction overt expression of emotion, and his male gender has another set of cultural expectations about behavior. He may be homosexual and belong to the gay culture, with the expectations and prejudices that accompany this orientation. His Roman Catholic upbringing adds another cultural layer that may contribute to his guilt, conflict, and confusion. It is easy to see how all of these multicultural influences provide the complex context that will impact his ability to resume his life in a healthy, productive way.

To diagnose and treat mental illness effectively, the APPN considers ethnicity, religion, race, class, cultural identity, cultural explanations of illness, and the cultural elements of the relationship between the individual and clinician. It is not possible to have extensive knowledge about many cultures, but a working knowledge of the backgrounds of those who most often seek treatment is essential. However, generalizations about another's culture do not tell us how to work with individuals. For example, knowing that those from a Hispanic culture often tend to somatize conflicts does not inform us about how to work with a Hispanic woman who hears the voice of her dead husband. It is highly likely that she may not be psychotic but is instead grieving according to acceptable cultural norms. Allowing time and support may be more appropriate than prescribing an antipsychotic drug.

The DSM-5 includes cultural formulation with interview questions designed for assessing information about the impact of a person's social and cultural context and the person's perceptions of the problem. This is a very helpful resource for mental health clinicians for effective assessment and clinical management and helps to establish rapport and engage the patient. In addition, a glossary of cultural concepts of distress is included in the DSM-5 Appendix, which illustrates syndromes in selected cultures. These syndromes illustrate the idiosyncratic nature of emotional concepts for various cultures; that is, it is how the culture perceives the emotional and bodily experiences that determines the characteristics and expressions of the disorder.

Recent cross-cultural research reveals that culture not only determines what is considered a psychiatric disorder but that neurobiological mechanisms linked to stress and trauma vary across cultures (Liddell \& Jobson, 2016). For example, higher cortisol levels following a stressful event have been found in Chinese children compared with children from the United States (Doan et al., 2017) and in Brazilian older adults compared with Canadian older adults (Souza-Talarico, Plusquellec, Lupien, Fiocco, \& Suchecki, 2014). This is important because relationships and culture shape not only our psychology but our biology. Research has also found that people from different cultural backgrounds vary in their interoceptive awareness (awareness of bodily states) (Ma-Kellams, 2014). Our bodily awareness, past experiences, and surroundings create emotions. Thus, emotions are constructed and predicted by the brain in the moment and learned in a relational context with the embedded values and norms of a particular culture (Barrett, 
2017). One's perception about the world is shaped by predictions the brain is making about physical autonomic states; thus, we are deeply interacting with each other on a visceral level. Assisting the patient in labeling and identifying and exploring emotions without preconceived assumptions about what the person is feeling is essential in all psychotherapy.

If the APPN is unfamiliar with a particular person's culture, consultation may be in order. It is also important to research that culture and to ask the patient about his or her own experience. Asking the person of a culture different from yours how he or she feels about working with you is respectful and opens up a dialogue about the experience for the patient. It is okay to tell the patient that you may make mistakes about his or her culture and experience and to ask the person to let you know if you do. For people of color who come to a White therapist or vice versa, racial differences often are "the elephant in the living room" and should be addressed to enable the person to stay in treatment. Asking out of a genuine curiosity and admitting ignorance are collaborative and reduce the power imbalance in the relationship by allowing the patient to teach us. For example, one young Black woman who came to therapy for depression explained how she had experienced prejudice, and implicit in this communication was her concern that her White therapist might be prejudiced, too. Through acknowledging ignorance about the experience of prejudice and exploring her feelings and experiences, the therapist and patient deepened their understanding of her fears about therapy as a forum in which she might be judged. This strengthened the therapeutic alliance and connection, which allowed her to remain in treatment.

\section{MENTAL ILLNESS}

According to Luhrmann (2000), a cultural anthropologist, there are traditionally two frameworks for understanding mental illness. One framework is the psychodynamic approach, originally based on Freud's theoretical speculations, but that has evolved into many other frameworks. This model attributes mental illness more or less to environmental and psychosocial problems (i.e., nurture). In contrast, the biophysiological model attributes mental illness to chemical imbalance (i.e., nature). The latter framework attributes mental illness to an imbalance of neurotransmitters in the brain, and the answer lies in correcting these imbalances, largely through medication. This model has revolutionized psychiatry and has been dominant since the 1950s, when phenothiazines were discovered with great excitement for the treatment of those with chronic mental illness or psychosis.

How changes in neurotransmitters produce symptoms has been an intense focus of investigation, beginning in the 1990s with the "decade of the brain." These studies are based on the underlying premise that mental illness is a "brain disease" and should be treated as any other illness. This idea has been embraced by mental health providers and drug companies, as well as those diagnosed with a psychiatric disorder. However, a seminal research study found that this belief actually increases rather than decreases stigma and that people thought to have a brain disease are treated more harshly (Mehta \& Farina, 1997). Perhaps diagnosing a person with a psychiatric disorder as "brain diseased" sets the person apart and further marginalizes the person as an "other." Stigma toward those with psychiatric disorders can be reduced through deepening our understanding of the effect of the environment on brain functioning. This knowledge may help to change the conversation from what is wrong with this person to what has happened to this person. 
Both genetic vulnerability and environmental influences play significant roles in the development of mental illness. The term epigenetics has been coined to describe this interplay, that is, the environment selects, signals, modifies, and regulates gene activity. Heritable differences in gene expression are now thought to be not the result of DNA sequencing but the effects of the encryption of experience that can be transmitted and alter behavior over generations. Genetic, biological, traumatic, and social factors interact, and this complex interplay shapes thinking, feelings, and behavior.

The stress diathesis model of psychiatric disorders has evolved from the recognition that genetics (diathesis/nature) and environment (stress/nurture) both contribute to the development of psychiatric disorders (Hankin \& Abela, 2005; Smoller, 2016). That is, for a person who has a genetic vulnerability and encounters significant early life stressors such as childhood trauma or neglect, loss, or viruses, the expression of the gene for the development of the psychiatric disorder most likely will be triggered. Evidence suggests that this is a result of changes in DNA through the process of methylation (Jiang, Postovit, Cattaneo, Binder, \& Aitchison, 2019). Methyl groups affix genes that govern the production of stress hormone receptors in the brain and this prevents the brain from regulating the response to stress. Parental nurturing mediates this epigenetic response; however, in the absence of parental nurturing, regulatory and attention problems result.

Two psychiatric disorders that are thought to be strongly heritable, schizophrenia and bipolar disorder, are now thought to share epigenetic roots. Significant epigenetic chemicals were found in the genome of 22 pairs of identical twins diagnosed with either schizophrenia or bipolar disorder (National Institute of Mental Health [NIMH], 2009). That is, the twins had identical DNA but significant differences were noted in the gene activity caused by their environment. This is strong evidence that supports the hypothesis that epigenetic mechanisms may drive even those psychiatric disorders considered most heritable (Coghlan, 2011). In addition, genetically identical twins are 50\% concordant for developing schizophrenia, which means that $50 \%$ of the variance is attributed to environmental or other nongenetic contributions (MacDonald \& Schulz, 2009). Some psychiatric disorders such as PTSD, reactive attachment disorder, acute stress disorder, and adjustment disorders are identified in the DSM-5 as trauma-related disorders and are triggered by exposure to extreme stress in people who otherwise may not be vulnerable (APA, 2013).

Animal and human studies strongly indicate that genetic factors of stress reactivity and greater physiological reactivity to stressful events may predispose one to a psychiatric disorder (Smoller, 2016). Those who have a stronger, more persistent response to stressors tend to withdraw from stressful situations and have internalizing traits. These people may be inhibited and more fearful, thus predisposing the person to anxiety and depressive disorders. Likewise, those whose temperament tends toward externalizing traits may be predisposed to develop psychopathology with symptoms of impulsivity, aggressiveness, and attentional difficulties. Caregivers who are not able to mediate arousal for their offspring with either of these traits are likely to exacerbate difficulties with affect and self-regulation that may lead to psychopathology (Schore, 2019).

Telomeres, DNA protein structures, have been found to be shortened in the presence of trauma. Telomere length is associated with the production of destructive radicals and molecules, chronic inflammation, co-occurring psychiatrc disorders, and a shorter life expectancy. A large meta-analysis found that PTSD was associated with shorter telomeres across gender (Li, Wang, Zhou, Huang, \& Li, 2017). Research suggests that childhood adversity affects our genes (Papale, Seltzer, Madrid, Pollak, \& Alisch, 2018). Gene expressions and salivary samples compared 22 girls who experienced high stress with those with normative stress. The high-stress group had more behavioral 
problems and 122 differentiated methylated genes. These genetic findings point to the transgenerational transfer of trauma from the first generation of trauma survivors to the second and further generations of offspring.

\section{Adverse Life Experiences}

It is now well established that adverse life experiences are associated with a wide range of psychiatric disorders and medical problems (Felitti \& Anda, 2010; Hughes et al., 2017; Suglia et.al., 2018). Felitti's (1998) seminal study of the long-term sequelae of adverse childhood experiences (ACE) for 17,421 middle class adults found a graded, positive relationship between $\mathrm{ACE}$ and significant heart disease, fractures, diabetes, obesity, unintended pregnancy, sexually transmitted diseases, depression, anxiety, sleep disorder, dissociative disorders, eating disorders, and alcoholism.

Approximately $70 \%$ of adults world wide experience at least one traumatic event within their lifetime (Kessler, 2017). Exposure using DSM-5 criteria is high (89.7\%); exposure to multiple traumatic event types is the norm (Kilpatrick et al, 2013). Childhood adversity is common. Globally, the World Health Organization (WHO, 2018) estimates that $25 \%$ of all adults have been physically abused as children. In the United States, $59 \%$ of adults report at least one adverse childhood experience (Shonkoff \& Gardner, 2012). One in ten children have experienced three or more ACEs and, in a number of states, the prevalence rate rises to one in seven (Sacks \& Murphy, 2018). Notably, a significant dose response relationship has been established: the higher the ACE score, the greater the likelihood of physical and mental illness. With an ACE score of 4 or more, the likelihood of chronic pulmonary lung disease increases 390 percent; hepatitis, 240 percent; depression 460 percent; attempted suicide, 1,220 percent (Fellitti \& Anda, 2010).

Children are particularly vulnerable to adverse events due to the plasticity of the developing brain; those who are brought up in a chaotic or non-nurturing environment suffer neurological consequences that are long-lasting and difficult to remediate (Shonkoff \& Garner, 2012). Toxic stress and ACE have been found to result in lifelong consequences for both psychological and physical health that affect behavior, economics, education, and health outcomes (Shonkoff, 2010). A longitudinal study of 2,232 twins, which controlled for genetic effects, found that children who experienced maltreatment by an adult or bullying by peers were more likely to report psychotic symptoms at age 12 than those who did not experience traumatic events (Arseneault et al., 2011). Similarly, a large meta-analysis of prospective and cross-sectional cohort studies found that ACE are associated with psychosis and that psychosis would be reduced by $33 \%$ if that risk factor were removed (Varese et al., 2012). In one large study of 30,000 children, almost every diagnosis of depression, anxiety, substance abuse, or eating disorders was comorbid with PTSD (Seng et al., 2005). The effects of trauma on the developing brain are likely to be cumulative, profound, and long-lasting; they transcend the diagnosis of PTSD and contribute to a wide range of physical, emotional, and social problems (Afifi, Mota, Dasiewicz, MacMillan \& Sareen, 2012; Nicholson et al, 2018; Porges, 2011; Schore, 2019; Teicher, 2012).

Toxic stress in early childhood also plays a role in the intergenerational transmission of disparities in health outcome (Braveman \& Barclay, 2009). Research supports the long-term negative sequelae related to the neurobiological responses to childhood stress and trauma (Jiang et al., 2019; Heins et al., 2011; Nicholson et al, 2018; Perry, 2001; Schore, 2012; Stien \& Kendall, 2006; Van Dam et al., 2012). These effects may then be inherited by subsequent generations: higher maternal ACE scores have been associated with measures of prenatal stress, autonomic nervous system reactivity, and psychopathology in her offspring (Esteves et al., 2020; Jones et al., 2019). 
The link between trauma and mental illness is complex and interactive. Numerous studies have found that adults receiving treatment for severe and persistent mental illness, substance abuse, eating disorders, anxiety, and depressive disorders are highly likely to be survivors of trauma, such as childhood sexual abuse, domestic or community violence, combat-related violence, or poverty (Brown et al., 2009; Chu, 2011; Danese et al., 2009; Read, 2010; Stien \& Kendall, 2006; Teicher, 2012). The majority of people served by public health mental health and substance abuse service systems have experienced repeated trauma since childhood and have been severely impacted by trauma (Grubaugh et al., 2011).

Racial trauma and the stress that results from danger related to real or perceived experiences of racial discrimination (Comas-Diaz, Hall, \& Neville, 2019) - has been identified as a precipitant of PTSD symptoms and is distinguished by the ongoing nature of its threat to the affected individual, such as through racial microagressions and vicarious experiences of bigotry (Helms, Nicolas, \& Green, 2012). Higher PTSD prevalence and severity among African American and Latinx adults has been linked to a greater frequency of perceived experiences of racism and discrimination in these populations (Sibrava et al., 2019). In one large systematic review and meta-analysis, self-reported experiences with racism were correlated with negative mental health outcomes, including depression, anxiety, and PTSD (Paradies et al., 2015).

Judith Herman (1992), in her seminal book Trauma and Recovery, states: "Traumatic events are extraordinary, not because they occur rarely, but rather they overwhelm the ordinary human adaptation to life" (p. 33). Findings in the wake of the World Trade Center disaster indicate that many people experienced significant symptoms, such as insomnia, irritability, general anxiety, vigilance, and impaired concentration, but did not qualify for a diagnosis of PTSD as defined by the DSM-IV-TR. People sought help for a wide variety of disabling clinical responses, but their pathologies were not reflected in the available diagnostic categories (Amsel \& Marshall, 2003). Similarly, in the absence of an identifiable Criterion A event (natural disasters, terrorist activities, war, incest, physical abuse, car accidents, or other major life-threatening events), those who experience PTSD symptoms as a result of racial trauma do not qualify for a DSM-5 PTSD diagnosis (Williams, Metzger, Leins, \& DeLapp, 2018).

van der Kolk (2014) says that while single-incident traumas may sometimes account for those diagnosed with posttraumatic stress disorder (PTSD), most adults who seek psychotherapy have had numerous traumatic events and suffer from a variety of psychological problems, many of which do not fall within this diagnostic category. Broadly speaking, these are problems such as aggression, self-hatred, dissociation, somatization, depression, distrust, shame, relationship problems, and affect regulation. Studies of children have found similar results; two thirds of children with documented abuse do not suffer from PTSD but do suffer from a variety of other psychiatric disorders, such as dissociative identity disorder, borderline personality disorder, depression, substance abuse, and attention deficit hyperactivity disorder (Fellitti \& Anda, 2010; Stien \& Kendall, 2006; Teicher et al., 2003; Teicher, 2012).

Adults who encounter everyday hardships are also at risk. A survey of 832 people from a primary care practice found that there were more PTSD symptoms for those who had suffered stressful life events than for those who had PTSD Criterion A events (Mol et al., 2005). Relatively common life events, such as the loss of a relationship or job, result in more PTSD symptoms than an actual or threatened death (Mol et al., 2005; Robinson \& Larson, 2010). Other studies confirm that even subthreshold PTSD results in significant functional impairments and a greater incidence of psychiatric disorders such as major depression, social anxiety, alcohol and drug use (McLaughlin et al., 2015; Mota et al, 2016). 
Common sense dictates that suffering any emotional or physical illness is disruptive, disturbing, and stressful. Accordingly, those who experience acute or chronic illness have been found to develop post-traumatic stress symptoms, including those admitted to the ICU (Parker et al., 2015); cancer survivors (Cordova, Riba, \& Spiegal, 2017); ischemic stroke patients (Stein et al., 2018); and people living with HIV (McLean \& Fitzgerald, 2016). Edmondson and colleagues found that those who developed PTSD due to their acute coronary event doubled their risk of recurrent future cardiac events and mortality (2012). Negative childbirth experiences, cesarean sections, and perinatal depression are risk factors for postnatal PTSD (Ayers, Bond, Bertullies, \& Wijma, 2016).

Pointedly, the experience of mental illness may in and of itself be regarded as a traumatic experience. For decades, studies have supported this possibility (McGorry et al., 1991; Meyer et al., 1999; Shaw et al., 1997). A diagnosis of PTSD plus depression and associated dissociative or borderline personality disorder appears to be a dose-response predictor for developing a chronic illness such as fibromyalgia, chronic fatigue syndrome, irritable bowel syndrome, chronic pelvic pain, and dysmenorrhea (Buskila \& Cohen, 2007). Moreover, after an exhaustive review of the literature on psychosis and trauma, Morrison and colleagues (2003) state, "... it does seem that at least a significant proportion of psychotic disorders do arise as a response to trauma and that PTSD-like symptoms can be developed in response to people's experience of psychotic disorders" (p. 347). The literature suggests that trauma is both a cause and an effect of medical and psychiatric pathologies.

The above research supports Shapiro's $(2001,2012,2018)$ expanded conceptualization of trauma from the Criterion A events for PTSD to include adverse life experiences that occur often and to most people, such as emotional neglect or indifference, humiliation, and family issues. For example, many childhood experiences, such as caregiver depression, chronic mother-infant misattunement, being bullied, chronic loneliness, significant loss, caregiver neglect, repeated separation from parents, betrayals, feeling stupid and humiliated in the classroom setting, abandonment, significant medical illness and procedures, relationship problems between parents, personality problems of parents, exposure to domestic violence, economic hardships, poverty, critical or negative comments from caretakers, social discrimination, prejudice, family instability, accidents, violence, frequent moves or changes of school, taking care of an alcoholic parent, and many other life events that impact the developing child, may contribute to mental health problems later in life. What is notable is that it is not just what has happened to the individual that is experienced as traumatic, but also what has not happened that should have happened, such as in situations of neglect and misattunement at critical periods of development.

The Substance Abuse and Mental Health Services Administration (SAMHSA) refers to trauma as:

.... experiences that cause intense physical and psychological stress reactions. It can refer to a single event, multiple events, or a set of circumstances that is experienced by an individual as physically and emotionally harmful or threatening and that has lasting adverse effects on the in-dividual's physical, social, emotional, or spiritual well-being (SAMHSA News, 2014).

The word "trauma" is used in this book to denote this expanded conceptualization inclusive of all events and situations that are experienced by the person as overwhelming and affect brain functioning through the interruption of information processing. This disruption in brain circuitry, regulatory systems, and information processing, results in the disturbing event or situation being stored dysfunctionally, sometimes 
affecting the person even decades later (Shapiro, 2018). It is a basic tenet of this book that such experiences are the basis of most psychopathology. Trauma is a response - a disconnection from oneself,- not an event (Mate, 2003; Porges, 2019). It is not so much what has happened to the person, but what happens inside the person. Healing trauma involves the reconnection with self and is always possible.

An individual's response and the long-term sequelae of a disturbing event are highly individualistic and depend on a multitude of factors, such as the person's age, developmental stage, coping skills, support system, cognitive deficits, preexisting neural physiology, and the nature of the trauma. It is not just the event itself that determines the long- and short-term effects of trauma, but the individual differences that the person brings to the situation. Traumatic experiences disrupt brain functioning as mediated by genetics, social support, age, development, and many other factors. Healthy functioning of the brain is reflected in the optimal integration and coordination of neural networks. Chapter 2 discusses the neurophysiological theory and research that provide the underpinnings for the psychotherapeutic framework for this book.

\section{A FRAMEWORK FOR PSYCHOTHERAPY PRACTICE}

The adaptive information processing (AIP) model, developed by Shapiro as an explanatory theory for EMDR, is a metamodel for understanding mental health and psychopathology, and provides direction for planning therapeutic interventions (Shapiro, 2018). AIP is a metamodel because mechanisms of action for all psychotherapy approaches can be explained by the neurophysiological underpinnings of AIP about how the brain works. AIP posits that normally information is taken in through the senses and connected adaptively to other memory networks so that storing and learning occur. There is thought to be innate self-healing in the brain and just as the body strives for homeostasis, so too does the brain through the regulation and processing of information through neural transmission. However, if something is experienced as overwhelming emotionally, brain processing is interrupted owing to the massive influx of hormones and neurotransmitters. It is as if our brain is saying: "Don't forget this, this is important!" These unprocessed experiences are considered to be the basis of the symptoms of many mental health problems and psychiatric disorders (Bergmann, 2020; Cozolino, 2017; Shapiro, 2018).

Once information processing is interrupted, the memory of the event becomes fragmented. The emotion related to the experience may become disconnected from the words to describe the event and/or the sound and/or physical sensations. Thus, the fragmented memory is not integrated but stored in the brain with each component existing in discrete units that are disconnected or dissociated from each other. The memory is stored largely in implicit or unconscious memory and is experienced as being in the present once triggered. For example, a woman who was raped 40 years ago might be triggered by having sex with her partner and feel as though the rape was happening all over again, or she may be anxious and fearful around certain places or people that remind her of the event and be unaware of why she is anxious and does not connect her current anxiety to the original experience. These reactions are not in her control but come from neural associations deep within memory networks that are not connected with the conscious mind. Consciousness is defined as our subjective experience of being aware and having access to information about the experience (Siegel, 2012). It is understandable that a person who has experienced multiple traumas may not be very conscious or living in the present. 


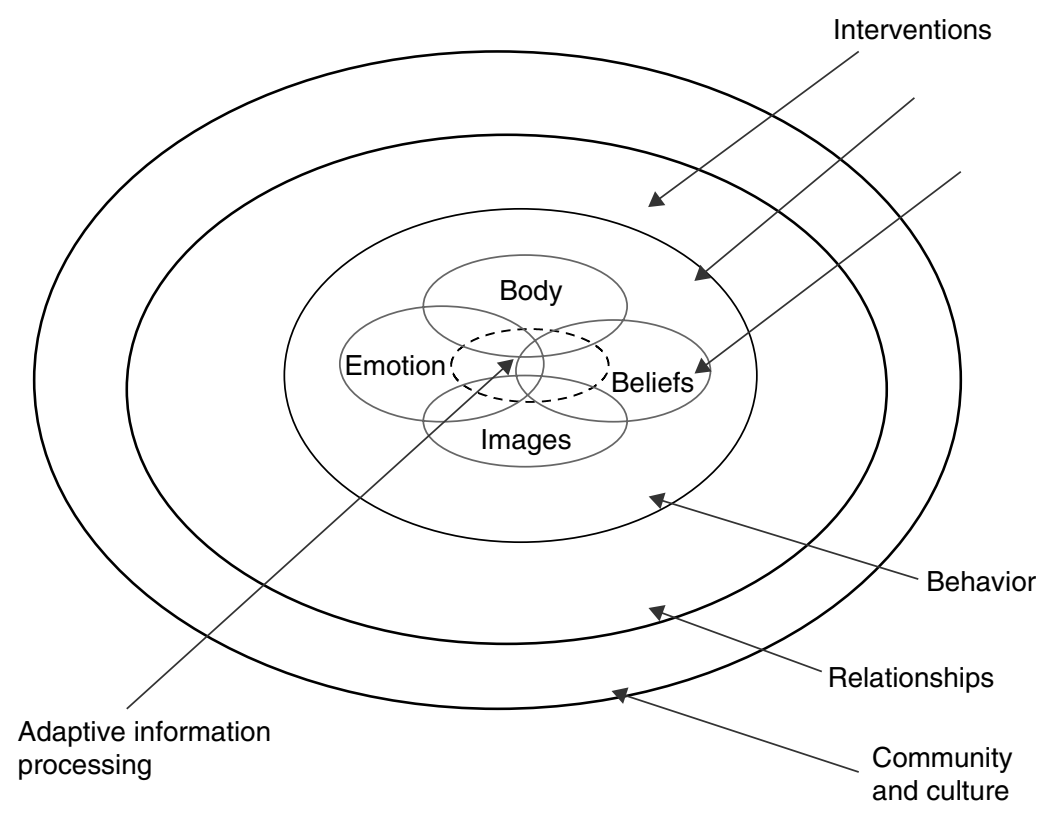

FIGURE 1.6 Where to target interventions.

Psychotherapy interventions can be designed to target any or all areas of the dissociated memory or experience-behavior, relationships, beliefs, the body, images, and/or emotions - to facilitate healing and promote neurophysiological harmony (see Figure 1.6). For example, the therapist using a CBT model would focus on the person's thoughts, beliefs, or behaviors; the therapist using a family therapy model would focus on the relationships and dynamics of the family; while the psychodynamic therapist would focus on emotions and thoughts assisting the person in deepening his or her understanding of how the past gets triggered and played out in the present. The EMDR therapist would target body, beliefs, images, and emotions in order to process trauma based on AIP. A change in any arena reverberates to all other dimensions for the overall purpose of facilitating healing and toward integration because all dimensions are interrelated and interconnected.

The treatment hierarchy framework for practice for this textbook based on AIP reflects a synthesis of research and theory developed by numerous clinicians and researchers (Briere \& Scott, 2013; Cozolino, 2017; Davis \& Weiss, 2004; Herman, 1992; Porges, 2011; Porges \& Dana, 2018; Schore, 2019; Shapiro, 2018; Siegel, 2012; Wheeler, 2011). To begin the healing process, the APPN assesses where to target interventions, taking into consideration the patient's culture and building on the strengths and resources the person already has. In general, the lower the patient is on Maslow's hierarchy of needs, the more active the therapist must be. For example, the patient who is abusing substances, hungry, and homeless must first have physiological needs and safety met first, and the APPN attends to these needs largely through case management strategies.

The treatment hierarchy illustrates an overarching framework for therapeutic aims that must be ensured before the person can move up the levels in the triangle (see Figure 1.7). The patient's physiological needs, such as diet, sleep, and exercise, are essential to a healthy emotional life and the work of psychotherapy. Nurses are knowledgeable about what constitutes a healthy lifestyle and this knowledge is invaluable to integrate into psychoeducation with patients in psychotherapy. Overall, resources must be procured and stabilization guaranteed before trauma can be processed, and then a vision can be developed of a possible future. The aim is toward integration of neural 


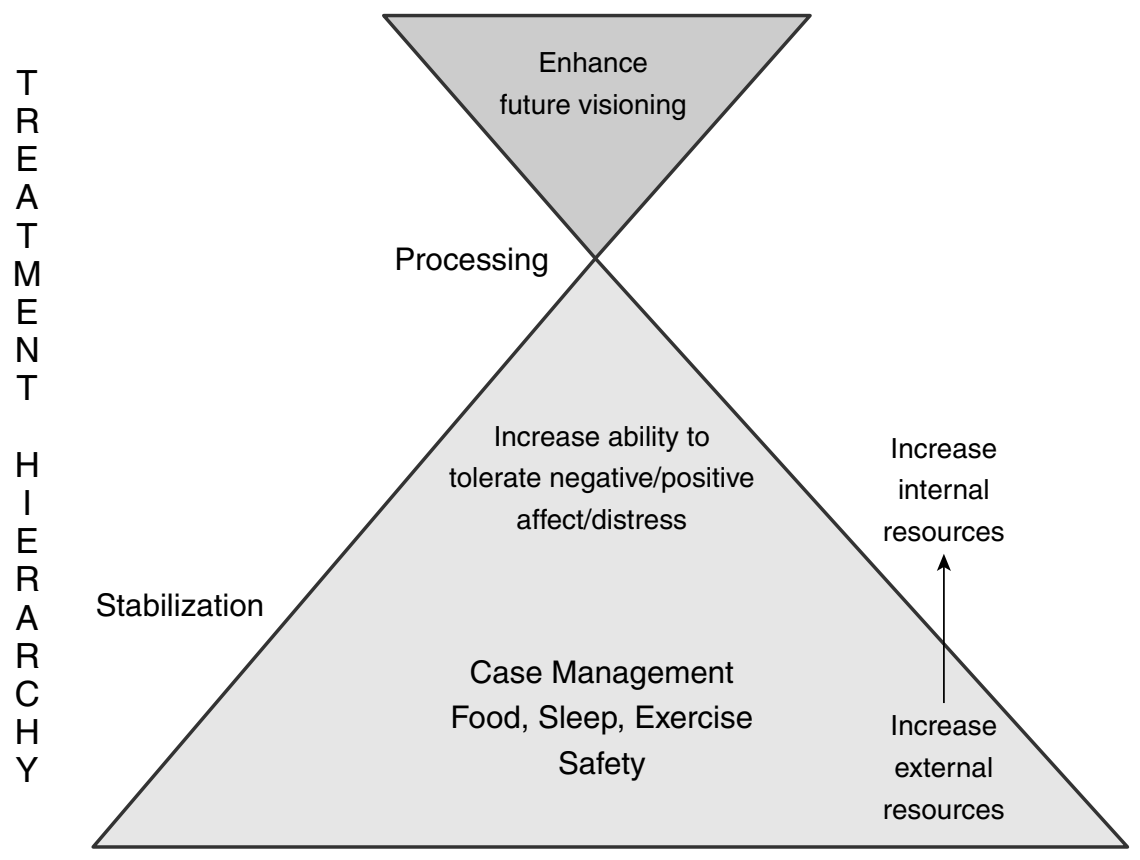

FIGURE 1.7 Treatment hierarchy framework for practice.

Source: Adapted from Davis, K., \& Weiss, L. (2004). Traumatology: A workshop on traumatic stress disorders. Hamden, CT: EMDR Humanitarian Assistance Programs.

networks, of memories, of oneself and relationships, and of the person's connection in the world. The patient's ability to process information is variable, with some patients needing more stabilization so that adaptive memory and experiences are created or are reinforced if present, while other patients may be able to process information and quickly move toward integration. Stabilization strengthens and/or widens the person's RZ so that daily functioning is improved. Integration through the phases of stabilization and processing or memory reconsolidation can be accomplished through the various psychotherapeutic approaches and techniques discussed in this book.

Some psychotherapeutic approaches may have more utility than others, depending on the person's state of need, resources already present, emotional development, past traumas, and support system as well as the expertise of the therapist. The therapist's thorough and accurate assessment, as discussed in Chapter 3, helps to formulate a plan to assist the person to move upward on the treatment hierarchy to the next stage. Although treatment is discussed as a stage model, it is not static in that there is some fluidity of movement. Frequently, patients take two steps forward and then one backward; that is, often after therapeutic gain, a period of anxiety, confusion, and/or depression follows. This is because emotion is a powerful agent of change and creates disruption (Damasio, 1999). Even a positive change may have a disorganizing effect on the brain and behavior because of the proliferation of synapses that occurs with new learning (Stien \& Kendall, 2006). This idea is supported by a developmental principle of all biological systems that "there can be no reorganization without disorganization" (Scott, 1979, p. 233). It is the therapist's responsibility to assist the person in understanding that the gains being made are often followed by increased sadness and anxiety. Explaining this to the person, keeping the overall plan and therapeutic aims in the foreground, and conveying hope is essential for the process and progress to continue. 
BOX 1.2 Selected Stabilization Strategies

- Through therapeutic relationship

- Bibliotherapy/role play

- Case management

- Cognitive behavioral therapy

- Community resiliency model skills

- Dialectical behavioral therapy

- Education about RZ

- Managing physiological arousal - imagery

\section{Stabilization}

Essential to all approaches discussed in this book is providing for safety and increasing resources, if needed, to attain stabilization and a robust RZ. For positive psychotherapy outcomes, Norcross and Lambert state to "begin by leveraging the patient's resources and self-healing capactities" (2019, p. 13). Resources might include a person's positive memories of past experience, spiritual beliefs, the availability of nurturing and caring people, a sense of inner strength or a belief in oneself, and coping strategies. Techniques used during the stabilization stage are sometimes referred to as case management or supportive psychotherapy but may also include any of the strategies included in Box 1.2 and community resiliency model (CRM) skills, included in Chapter 11. Competency in case management includes the ability of the therapist to garner the necessary environmental resources on behalf of the patient and requires an active approach on the part of the therapist. Setting limits, educating, connecting the person to community resources, supporting the patient's ego functions, and assisting the patient in managing emotions are key to successful outcomes. A worksheet for treatment and case management strategies is included in Appendix 1.4 from Seeking Safety: A Treatment Manual for PTSD and Substance Abuse, by Najavits (2002); it is an excellent resource for case management.

Stabilization strategies assist the person to be better able to make state changes, that is, to change one's present physiology in order to function more effectively in the moment. Crucial in case management is the ability of the therapist to assess regressive and adaptive shifts in ego functioning and to recognize conflict to help the person to manage anxiety. Although the therapist may understand what is happening for the patient dynamically, this does not need to be interpreted to the person. Accurate assessment of where the person is in the change process is essential. A stage of change model is helpful in determining where the therapist needs to aim interventions. This is especially useful for interventions aimed at behavioral change. Stages of change are discussed in Chapter 9.

Along with behavioral change and shoring up external resources, if needed, internal resources often need to be increased before processing. Internal resources are less tangible than external resources, and include the person's ability to manage positive and negative emotions. Indicators that the person has sufficient internal resources include the person's ability to self-soothe, to demonstrate adequate impulse control, to identify stressful triggers, to regulate moods, and to communicate honestly. In general, the patient's resources and the traumas experienced need to be balanced. For the person who has a history of many adverse life experiences without positive memories 


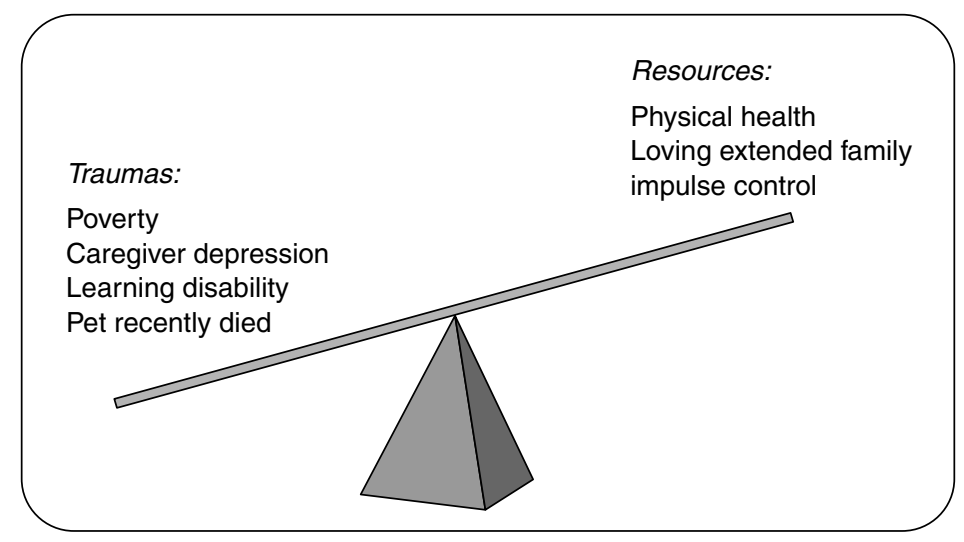

FIGURE 1.8 Trauma and resource balance.

or experiences, more resources may be needed to manage the deleterious effect of these experiences on functioning to enhance the RZ. For example, a 6-year-old, learning-disabled boy, whose dog recently died, started to wet his bed nightly and was brought to the clinic by his mother. He lived with his mother, who was single, chronically depressed, and had significant economic problems. Figure 1.8 illustrates that more resources may need to be developed for this child to counteract the imbalance between his traumas and resources or strengths. The assessment of adverse experience and resource balance is based on a comprehensive history and assessment as outlined in Chapter 3.

A stabilization checklist is included in Appendix 1.5 to help the clinician determine whether adequate stabilization has been achieved. The person does not need to meet all the criteria on this list before processing and the therapist's clinical judgment is essential in order to determine appropriate strategies. Sometimes the instability is driven by the trauma and once the traumatic memory has been processed, symptoms will dissipate. Specific strategies designed to widen or strengthen the RZ are delineated in the psychotherapy approaches included in this textbook.

\section{Processing}

After stabilization has been achieved, the person is ready to move to the next stage of processing. As represented toward the top of the treatment hierarchy in Figure 1.7, processing reflects access to all dimensions of memory: behaviors, affect, sensations, cognitions, and beliefs associated with the trauma (Shapiro, 2018). Processing usually involves assisting the person in constructing a narrative through the exploration of the meaning of significant adverse life experiences and traumas that impair functioning. Changes in physical and emotional responses occur as components of the dysfunctional memory are integrated with other, more adaptive networks. In contrast to state changes that occur in stabilization, processing creates trait changes, that is, enduring relationship and personality changes (Shapiro, 2018). The therapist assists the person in processing using the models and techniques discussed throughout this textbook. Some psychotherapy approaches involve components of stabilization as well as processing such as psychodynamic psychotherapy and EMDR therapy; others such as imaginal or in vivo exposure are designed primarily for processing a specific traumatic event. Processing strategies are included in Box 1.3. 
BOX 1.3 Selected Processing Strategies

- Through therapeutic relationship

- Psychodynamic psychotherapy

- Imaginal or in vivo exposure

- Cognitive processing

- Somatic processing

- Eye movement desensitization and reprocessing

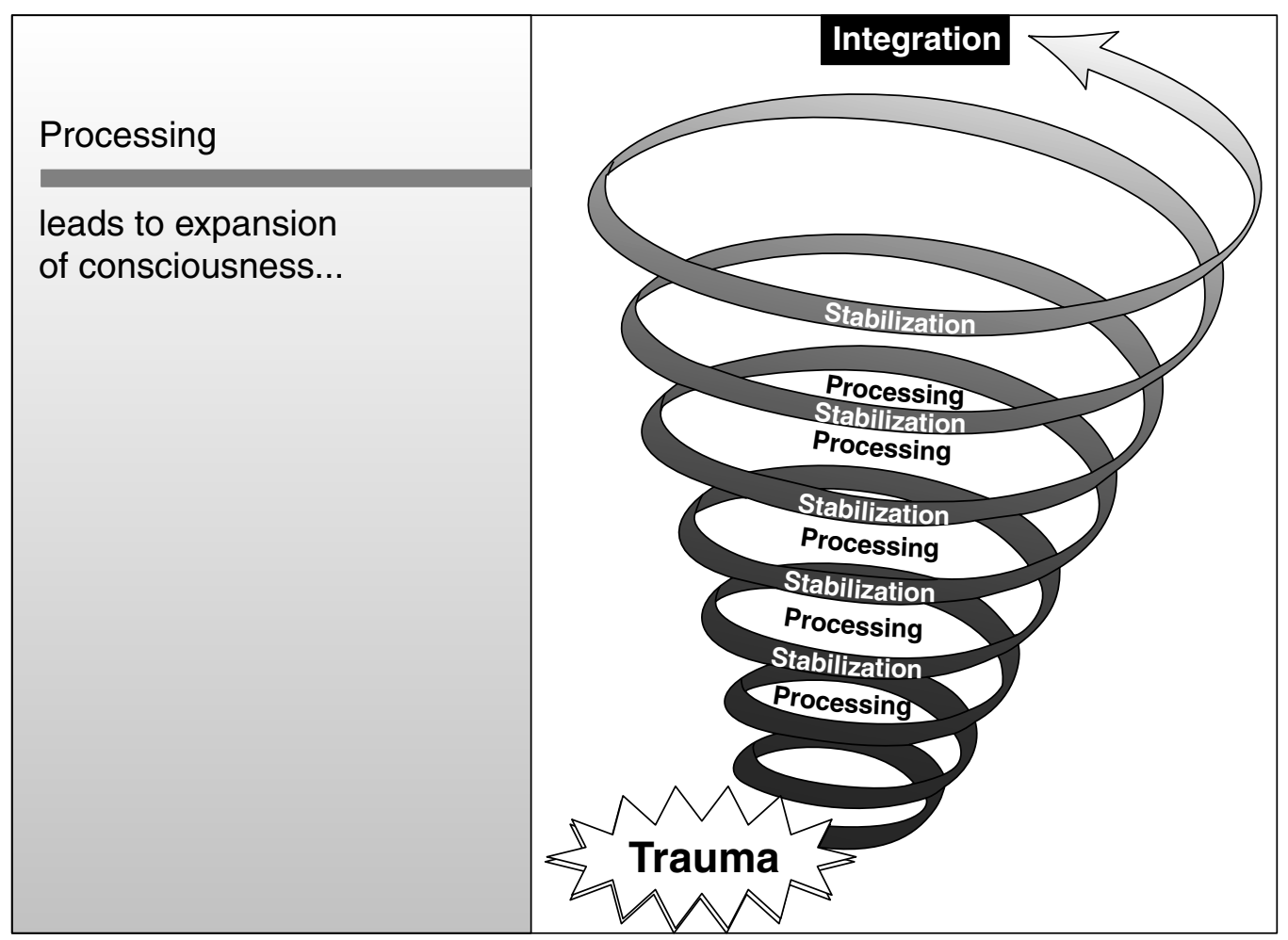

FIGURE 1.9 Spiral of treatment process.

Communication techniques can also facilitate stabilization or processing and are discussed in Chapter 4.

Processing is based on the idea that humans have an inherent information processing system that usually integrates experiences to a physiological adaptive state in which information can be taken in, and learning will occur (Shapiro, 2012, 2018). Memory is stored in neural networks that are linked together and organized around early events with associated emotions, thoughts, images, and sensations. Healthy functioning is reflected in the optimal integration and coordination of these neural networks, and this occurs through processing information. The neurophysiology underlying processing is discussed in Chapters 2 and 7.

Clinically, processing has been achieved once relationships are adaptive, work is productive, self-references are positive, there are no significant affect changes, affect is 
proportionate to events, and there is congruence among behavior, thoughts, and affect. A processing checklist is included in Appendix 1.6 to assist the therapist in determining whether processing has led to adaptive change. Periods of processing are usually followed by periods of destabilization, and the treatment process often looks more like a spiral alternating with interventions aimed at stabilization and then processing (Figure 1.9). Ongoing assessment and attunement to the person's therapeutic process are important in order to monitor progress and plan treatment strategies. The psychotherapeutic relationship is the vehicle for therapeutic change with the therapist's presence serving to stabilize the person and provides the foundation needed to assist the integration of dimensions of memory and all parts of the self at deeper levels of understanding. The therapeutic relationship may also facilitate processing as dimensions of earlier significant relationships through transference are triggered and reworked in the present. Empowerment and autonomy are fostered as the person moves toward envisioning and planning for the future.

\section{CASE EXAMPLE}

Ms. A is a 26-year-old married woman who works as a costume designer and seamstress for a theater company. She has been in psychotherapy numerous times since the age of 13 for past psychiatric diagnoses of major depressive disorder, bipolar II, panic disorder, and anorexia nervosa. She reports numerous psychosomatic complaints including frequent stomachaches, irritable bowel syndrome, acid reflux, headaches, restless legs syndrome, and generalized pain as well as cold chills all over her body. Her reason for seeking treatment was her anxiety and insomnia related to her loud, annoying neighbors at her condo. Ms. A's early history involved significant attachment problems with both her mother and father abusing drugs, and subsequently Ms. A was taken away from her parents and into custody by her aunt when she was 2,years,old. Ms. A said her aunt favored her own biological daughter and neglected her throughout her childhood. Her adult trauma history included two previous car accidents. On intake, she scored 63 on the Spielberger Trait Anxiety Scale; 22 on the Beck Depression Inventory; and 27 on

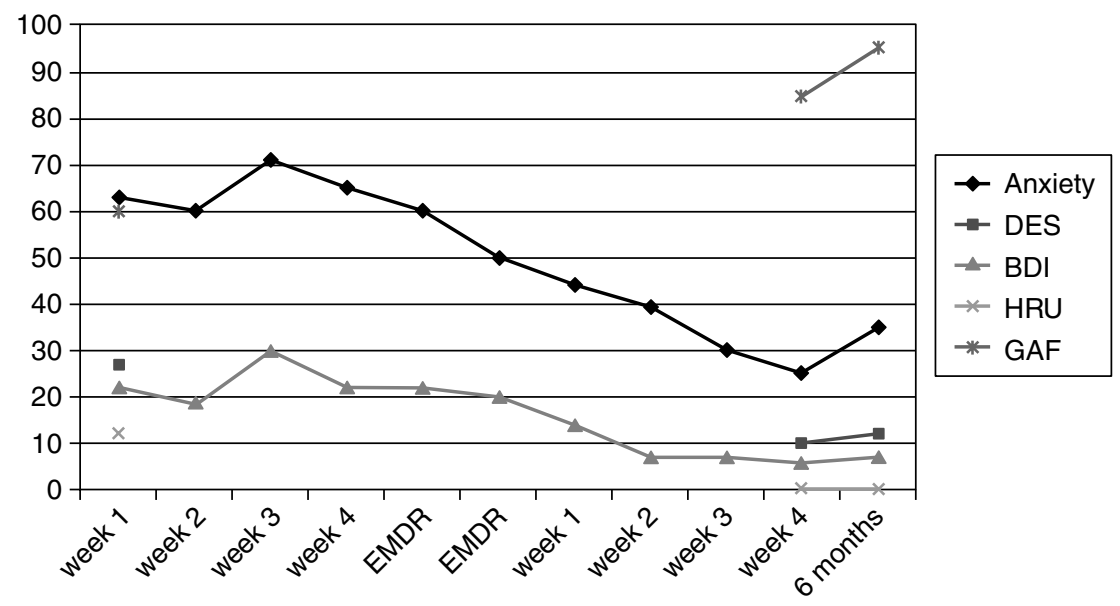

FIGURE 1.10 Ms. A's psychotherapy outcomes.

Anxiety, Spielberger trait anxiety scale; BDI, Beck depression inventory; DES, dissociative experiences scale; GAF, global assessment of functioning; HRU, health resource utilization 
the Dissociative Experiences Scale. All scores were significant for anxiety, depression, and dissociation, respectively.

Because Ms. A had significant attachment problems by history, discomfort with her body and physical sensations, difficulty self-soothing, some dissociation, difficulty tolerating negative emotions, and inadequate trust of others, her RZ was quite narrow. Ms. A perceived her current problem with her neighbors as an unmanageable crisis. The RZ was explained to Ms. A: "There is a biological basis for the symptoms you are experiencing. When the nervous system is in balance we feel like our best self and are in our resilient zone. It sounds like you have been under a lot of stress lately and are being triggered by your neighbors' noise, which has knocked you out of your resilient zone so you are feeling anxious and depressed. It would be helpful to begin first with working on some strategies to help stabilize your nervous system. Would that be okay?" The beginning phase of therapy focused on developing a therapeutic alliance, teaching Ms. A stabilization strategies that were practiced in sessions such as imagery of safe/calm place (Appendix 1.7) and container exercises (included in Appendix 1.8); the Fraser Table Technique, which is an imagery exercise designed to facilitate knowledge of various parts of the self (Fraser, 1991); attachment imagery exercises (Steele, 2007); somatic awareness where she perceived feelings in her body (tracking in Chapter 11); and building resources outside therapy by increasing activities she liked such as regular exercise and yoga classes. Prozac $20 \mathrm{mg}$ was also prescribed to help Ms. A manage her anxiety. Adequate resources must be present in order to ensure safe processing. EMDR therapy was integrated with these stabilization strategies to process her traumas. Ms. A was seen over a 6-month period.

At termination, Ms. A's scores on all measures showed significant improvement (see Figure 1.10). Ms. A's creativity, visual imaging skills, and humor were great assets to her in our work together. Less tangible outcomes than the reported quantitative data were qualitative outcomes, which included an integrative narrative about herself and her aunt that included a recognition of the impact of her past history; greater ability to express herself and advocate for her own needs; better emotional and physical self-regulation; a sense of security about herself and others; and greater access to full expression of emotion. Her somatic complaints greatly decreased and as illustrated in the graph in Figure 1.10, she did not need or seek medical care for her many illnesses during the course of her psychotherapy treatment in contrast to the 6 months prior to therapy when she had sought help from her primary care provider a total of 12 times. She appeared more robust and stronger at termination, stating that she had never felt this good before.

\section{CONCLUDING COMMENTS}

Psychotherapy has been identified as an important competency that all APPNs must achieve (ANA, 2013; National Panel for Psychiatric Mental Health NP Competencies, 2003; Wheeler \& Haber, 2004). Nurses who are beginning graduate study in psychiatric nursing and expanding their roles to become psychotherapists have unique resources and challenges. The holistic paradigm inherent in nursing provides the context and a compass for psychotherapy practice, whereas the models of psychotherapy presented in this textbook provide the vehicle, anchored in the mooring of the healing nurse-patient relationship. Providing a context rich in resources enables further growth toward healing and wholeness. An appropriate metaphor is that of building a house with the foundation and frame necessary for support before furnishing and decorating. Providing for safety and stabilization by strengthening external and internal resources facilitates resilience so that healing can occur. 
Adverse experiences have the potential to abort the wholeness of the brain, interfering with information processing, and this disruption and dysregulation, sometimes in tandem with neurobiologically encoded genetic vulnerabilities, are the basis for many mental health problems and psychiatric disorders. Psychotherapy assists in reintegration of neural networks that have become dysregulated or disconnected, enhancing the development of the brain so that continued growth and healing can occur. This framework is based on neurophysiology embedded in a holistic paradigm in that psychotherapy restores the harmony, balance, connection, and integration of neural networks on a cellular level, which is reflected in deeper connections with oneself and others. The neurophysiological basis for this model is discussed in Chapter 2.

\section{DISCUSSION QUESTIONS}

1. In light of Benner's model, where do you see yourself in relation to your past practice of nursing, and where are you now in your nurse psychotherapy practice?

2. How does your choice of intervention affect the outcome of treatment?

3. How can a person be healed and still have a diagnosed psychiatric disorder? How is curing different from healing? How do you know when healing has occurred?

4. Discuss a time when you and your patient had a different perception of health and illness and what this experience was like for you. How was this worked out then, and what would you do differently now?

5. Discuss how your self-understanding may affect your work with your patient. How has your own growth changed since you first began to work with others? Include your thoughts about how your prior practice as a nurse can be a help or hindrance to your practice as a psychotherapist.

6. What factors in your life led you to a nurse psychotherapist's role?

7. Discuss relationship and resilience and give a clinical example of each from your past nursing practice.

8. Describe a patient you have worked with, explain the person's traumas and resources, and discuss in general the priorities for treatment using the practice treatment hierarchy.

\section{REFERENCES}

Afifi, T. O., Mota, N. P., Dasiewicz, P., MacMillan, H. L., \& Sareen, J. (2012). Physical punishment and mental disorders: Results from a nationally representative US sample. Pediatrics, 130, 184-192. doi:10.1542/peds.2011-2947

American Hospital Association. (2019). For the 17th year in a row, nurses top Gallup's poll of most trusted profession. Retrieved from https://www.aha.org/news/insights-and-analysis/201901-09-17th-year-row-nurses-top-gallups-poll-most-trusted-profession

American Nurses Association. (2013). Psychiatric-mental health nursing: Scope and standards of practice. Washington, DC: Author.

American Nurses Credentialing Center. (2020). Psychiatric mental health nurse practitioner eligibility \& instructions. Silver Spring, MD: American Nurses Credentialing Center, American Nurses Association. Retrieved from https:/ /www.nursingworld.org/our-certifications / psychiatric-mental-health-nurse-practitioner/

American Psychiatric Association. (2013). Diagnostic and statistical manual of mental disorders (5th ed.). Washington, DC: American Psychiatric Publishing.

Amsel, L., \& Marshall, R. D. (2003). Clinical management of subsyndromal psychological sequelae of the 9/11 terror attacks. In S. Coates, J. Rosenthal, \& D. Schechter (Eds.), September 11: Trauma and human bonds (pp. 75-97). Hillsdale, NJ: Analytic Press. 
Arseneault, L., Cannon, M., Fisher, J., Polanczyk, G., Moffitt, T. E., \& Caspi, A. (2011). Childhood trauma and children's emerging psychotic symptoms: A genetically sensitive longitudinal cohort study. American Journal of Psychiatry, 168, 65-72. doi:10.1176/appi.ajp.2010.10040567

Ayers, S., Bond, R., Bertullies, S., \& Wijma, K. (2016). The aetiology of post-traumatic stress following childbirth: a meta-analysis and theoretical framework. Psychological Medicine, 46(6), 1121-1134.

Balsam, R., \& Balsam, A. (1974). Becoming a psychotherapist: A clinical primer. Boston, MA: Little Brown.

Barrett, L. F. (2017). How emotions are made: The secret life of the brain. Boston, MA: Houghton Mifflin Harcourt.

Beeber, L. (1995). The one-to-one nurse patient relationship in psychiatric nursing: The next generation. In C. A. Anderson (Ed.), Psychiatric nursing 1974-1994: A report on the state of the art (pp. 9-36). St. Louis, MO: Mosby Year Book.

Benner, P. (1984). From novice to expert. Menlo Park, CA: Addison-Wesley.

Bergmann, U. (2020). Neurobiological foundations for EMDR practice (2nd ed.). New York, NY: Springer Publishing Company.

Bourne, E. J. (2015). The anxiety and phobia workbook (6th ed.). Oakland, CA: New Harbinger.

Braveman, P., \& Barclay, C. (2009). Health disparities beginning in childhood: A life-course perspective. Pediatrics, 124(Suppl. 3), 163-175. doi:10.1542/peds.2009-1100d

Briere, J., \& Scott, C. (2013). Principles of trauma therapy: A guide to symptoms, evaluation, and treatment (2nd ed.). Thousand Oaks, CA: Sage.

Brown, D. W., Anda, R. F., Tiemeier, H., Felitti, V. J., Edwards, V. J., Croft, J. B., \& Giles, W. H. (2009). Adverse childhood experiences and the risk of premature mortality. American Journal of Preventive Medicine, 37, 389-396. doi:10.1016/j.amepre.2009.06.021

Bunkers, S. S. (2009). The power and possibility in listening. Nursing Science Quarterly, 23(1), 22-27. doi:10.1177/0894318409353805

Burke, B. T., Miller, B. F., Proser, M., Petterson, S. M., Bazemore, A. W., \& Phillips, R. L. (2013). A needs-based method for estimating the behavioral health staff needs of community health centers. BMC Health Services Research, 13, 245. doi:10.1186/1472-6963-13-245

Buskila, D., \& Cohen, H. (2007). Comorbidity of fibromyalgia and psychiatric disorders. Current Science, 11(5), 333-338. doi:10.1007/s11916-007-0214-4

Chu, J. A. (2011). Rebuilding shattered lives: Treating complex PTSD and dissociative disorders (2nd ed.). New York, NY: John Wiley \& Sons.

Cloos, J.-M., \& Ferreira, V. (2009). Current use of benzodiazepines in anxiety disorders. Current Opinion in Psychiatry, 22(1), 90-95. doi:10.1097/YCO.0b013e32831a473d

Coghlan, A. (2011). Epigenetic clue to schizophrenia and bipolar disorder. New Scientist. Retrieved from http://www.newscientist.com/article/mg21128323.400-epigenetic-clue-toschizophrenia-and-bipolar-disorder.html

Comas-Diaz, L. Hall, G. N., \& Neville, H. A. (2019). Racial trauma: Theory, research, and healing: Introduction to the special issue. American Psychologist, 74(1), 1-5.

Cordova, M. J., Riba, M. B., \& Spiegel, D. (2017). Post-traumatic stress disorder and cancer. The Lancet. Psychiatry, 4(4), 330-338.

Cozolino, L. (2017). The neuroscience of psychotherapy: Healing the social brain (3rd ed.). New York, NY: W. W. Norton.

Dahlgaard, J., Jorgensen, M. M., van der Velden, A. M., Sumbundu, A., Gregersen, N., Olsen, R. K., . . Mehlsen, M. Y. (2019). Mindfulness, health, and longevity. In S. I. S. Rattan \& M. Kyriazis (Eds.), The science of hormesis in health and longevity (pp. 243-256). San Diego, CA: Academic Press.

Damasio, A. (1999). The feeling of what happens: Body and emotion in the making of consciousness. New York, NY: Harcourt Brace.

Danese, A., Moffit, T. E., Harrington, H., Milne, B. J., Polanczyk, G., Pariante, C. M., . . Caspi, A. (2009). Adverse childhood experiences and adult risk factors for age-related disease: Depression, inflammation, and clustering of metabolic risk markers. Archives of Pediatrics $\mathcal{E}$ Adolescent Medicine, 163(12), 1135-1143. doi:10.1001/archpediatrics.2009.214

D'Antonio, P., Beeber, L., Sills, G., \& Naegle, M. (2014). The future in the past: Hildegard Peplau and interpersonal relations in nursing. Nursing Inquiry, 21, 311-317. doi:10.1111/nin.12056

Davis, K., \& Weiss, L. (2004). Traumatology: A workshop on traumatic stress disorders. Hamden, CT: EMDR Humanitarian Assistance Programs. 
De Bellis, M. (2001). Developmental traumatology: The psychobiological development of maltreated children and its implications for research treatment, and policy. Development and Psychopathology, 13, 539-564. doi:10.1017/s0954579401003078

Delaney, K. R., Drew, B. L., \& Rushton, A. (2019). Report on the APNA national psychiatric mental health advanced practice registered nurse survey. Journal of the American Psychiatric Nurses Association, 25(2), 146-155. doi:10.1177/1078390318777873

Doan, S. N., Tardif, T., Miller, A., Olson, S., Kessler, D., Felt, B., \& Wang, L. (2017). Consequences of 'tiger' parenting: A cross-cultural study of maternal psychological control and children's cortisol stress response. Developmental Science, 20(3), e12404. doi:10.1111/desc.12404

Dossey, B., \& Keegan, L. (2013). Holistic nursing: A handbook for practice (6th ed.). Burlington, MA: Jones \& Bartlett.

Dracup, K., Conenwett, L., Meleis, A., \& Benner, P. (2005). Reflections on the doctorate of nursing practice. Nursing Outlook, 53, 177-182. doi:10.1016/j.outlook.2005.06.003

Drew, B., \& Delaney, K. R. (2009). National survey of psychiatric mental health advanced practice nursing: Development, process and findings. Journal of the American Psychiatric Nurses Association, 15, 101-110. doi:10.1177/1078390309333544

Edmondson, D., Richardson, S., Falzon, L., Davidson, K. W., Mills, M. A., \& Neria, Y. (2012). Posttraumatic stress disorder prevalence and risk of recurrence in acute coronary syndrome patients: A meta-analytic review. PLOS ONE, 7(6), e38915. doi:10.1371/journal.pone.0038915

Elklit, A., \& Blum, A. (2011). Psychological adjustment one year after the diagnosis of breast cancer: A prototype study of delayed post-traumatic stress disorder. British Journal of Clinical Psychology, 50, 350-363. doi:10.1348/014466510X527676

Esteves, K. C., Jones, C. W., Wade, M., Callerame, K., Smith, A. K., Theall, K. P., \& Drury, S. S. (2020). Adverse childhood experiences: Implications for offspring telomere length and psychopathology. The American Journal of Psychiatry, 177(1), 47-57.

Felitti, V. J., \& Anda, R. F. (2010). The relationship of adverse childhood experiences to adult medical disease, psychiatric disorders and sexual behavior: Implications for healthcare. In R. Lanius, E. Vermetten, \& C. Pain (Eds.), The impact of early life trauma on health and disease: The hidden epidemic (pp. 77-87). Cambridge, UK: Cambridge University Press. doi:10.1017/ CBO9780511777042.010

Felitti, V. J., Anda, R. F., Nordenberg, D., Williamson, D. F., Spitz, A. M., Edwards, V., . . Marks, J. S. (1998). Relationship of childhood abuse and household dysfunction to many of the leading causes of death in adults: The adverse childhood experiences (ACE) study. American Journal of Preventive Medicine, 14(4), 245-258. doi:10.1016/S0749-3797(98)00017-8

Foli, K. J., \& Thompson, J. R. (2019). The influence of psychological trauma in nursing. Indianapolis, IN: Sigma Theta Tau International.

Forchuk, C., Westwell, J., Martin, M. L., Azzapardi, W. B., Kosterewa-Tolman, D., \& Hux, M. (1998). Factors influencing movement of chronic psychiatric patients from the orientation of the working phase of the nurse-patient relationship on an inpatient unit. Perspectives in Psychiatric Care, 34, 36-45. doi:10.1111/j.1744-6163.1998.tb00998.x

Fraser, G. (1991). The dissociative table technique: A strategy for working with ego states in dissociative disorders and ego state therapy. Dissociation, 4(8), 205-213.

Gallup. (2020). Nurses continue to rate highest in honesty, ethics. Retrieved from https:/ / news. gallup.com/poll/274673/nurses-continue-rate-highest-honesty-ethics.aspx

Gilson, G., \& Kaplan, S. (2000). The therapeutic interweave in EMDR: Before and beyond: A manual for EMDR trained clinicians. New Hope, PA: EMDR Humanitarian Assistance Programs.

Grubaugh, A. L., Zinzow, H. M., Paul, L., Egede, L. W., \& Freuh, C. B. (2011). Trauma exposure and posttraumatic stress disorder in adults with severe mental illness: A critical review. Clinical Psychology Review, 31, 883-899. doi:10.1016/j.cpr.2011.04.003

Habibović, M., van den Broek, K., Alings, M., Van der Voort, P. H., \& Denollet, J. (2011). Posttraumatic stress 18 months following cardioverter defibrillator implantation: Shocks, anxiety, and personality. Health Psychology, 31(2), 186-193. doi:10.1037/a0024701

Hankin, B. L., \& Abela, J. (2005). Development of psychopathology: A vulnerability-stress perspective. Thousand Oaks, CA: Sage.

Heins, M., Simons, C., Lataster, T., Pfeifer, S., Vermissen, D., Lardinois, M., . . Myin-Germeys, I. (2011). Childhood trauma and psychosis: A case-control and case-sibling comparison across 
different levels of genetic liability, psychopathology, and type of trauma. American Journal of Psychiatry, 168(12), 1286-1294. doi:10.1176/appi.ajp.2011.10101531

Helms, J. E., Nicolas, G., \& Green, C. E. (2012) Racism and ethnoviolence as trauma: Enhancing profressional and research training. Traumatology, 18(1), 65-74.

Herman, J. (1992). Trauma and recovery. New York, NY: Basic Books.

Herrman, H., Stewart, D., Diaz-Grandos, N., Berger, E., Jackson, B., \& Yuen, T. (2011). What is resilience? The Canadian Journal of Psychiatry, 65(5), 258-265. doi:10.1177/070674371105600504

Horowitz, A. V. (1982). The social control of mental illness. New York, NY: Academic Press.

Hughes, K., Bellis, M. A., Hardcastle, K. A., Sethi, D., Butchart, A., Mikton, C., Jones, L., \& Dunne, M. P. (2017). The effect of multiple adverse childhood experiences on health: a systematic review and meta-analysis. The Lancet. Public Health, 2(8), e.356-e366.

Jennings, A. (2004). Models for developing trauma-informed behavioral health systems and traumaspecific services. Retrieved from http:/ / theannainstitute.org/MDT.pdf

Jiang, S., Postovit, L, Cattaneo, A., Binder, E. \& Aitchison K. (2019). Epigenetic modifications in stress response genes associated with childhood trauma. Frontiers in Psychiatry, 08, November. doi:10.3389/fpsyt.2019.00808

Jones, C. W., Esteves, K. C., Gray, S., Clarke, T. N., Callerame, K., Theall, K. P., \& Drury, S. S. (2019). The transgenerational transmission of maternal adverse childhood experiences (ACEs): Insights from placental aging and infant autonomic nervous system reactivity. Psychoneuroendocrinology, 106, 20-27.

Kessler, R. C., Aguilar-Gaxiola, S., Alonso, J., Benjet, C., Bromet, E. J., Cardoso, G., . . . Koenen, K. C. (2017). Trauma and PTSD in the WHO world mental health surveys. European Journal of Psychotraumatology, 8 (suppl. 5), 1353383.

Kilpatrick, D. G., Resnick, H. S., Milanak, M. E., Miller, M. W., Keyes, K. M., \& Friedman, M. J. (2013). National estimates of exposure to traumatic events and PTSD prevalence using DSM-IV and DSM-5 criteria. Journal of Traumatic Stress, 26(5), 537-547. doi:10.1002/jts.21848

Kronos. (2017). Employment engagement in nursing. Retrieved from https:/ / www.kronos.com/ about-us/newsroom/kronos-survey-finds-nurses-love-what-they-do-though-fatiguepervasive-problem

Lego, S. (1992). Biological psychiatry and psychiatric nursing in America. Archives of Psychiatric Nursing, 6, 147-150. doi:10.1016/0883-9417(92)90025-E

Lego, S. (1999). The one-to-one nurse-patient relationship. Perspectives in Psychiatric Care, 35(4), 4-22. doi:10.1111/j.1744-6163.1999.tb00591.x

Levine, S., Lalufer, A., Stein, E., Hamama-Raz, Y., \& Solomon, Z. (2009). Examining the relationship between resilience and posttraumatic growth. Journal of Traumatic Stress, 22(4), 282-286. doi:10.1002/jts.20409

Li, X., Wang, J., Zhou, J., Huang, P., \& Li, J. (2017). The association between post-traumatic stress disorder and shorter telomere length: A systematic review and metaanalysis. Journal of Affective Disorders, 218, 322-326. doi:10.1016/j.jad.2017.03.048

Li, Y., Cao, F., Cao, D., \& Liu, J. (2015). Nursing students' post-traumatic growth, emotional intelligence and psychological resilience. Journal of Psychiatric and Mental Health Nursing, 22, 326-332. doi:10.1111/jpm.12192

Liddell, B. J., \& Jobson, L. (2016). The impact of cultural differences in self-representation on the neural substrates of posttraumatic stress disorder. European Journal of Psychotraumatology, 7(1), 30464. doi:10.3402/ejpt.v7.30464

Livingston, J. D., \& Boyd, J. E. (2010). Correlates and consequences of internalized stigma for people living with mental illness: A systematic review and meta-analysis. Social Science Medicine, 71, 2150-2161. doi:10.1016/j.socscimed.2010.09.030

Luhrmann, T. M. (2000). Of two minds: An anthropologist looks at American psychiatry. New York, NY: Vintage Books.

MacDonald, A., \& Schulz, S. C. (2009). What we know: Findings that every theory of schizophrenia should explain. Schizophrenia Bulletin, 35(3), 493-508. doi:10.1093/schbul/sbp017

Ma-Kellams, C. (2014). Cross-cultural differences in somatic awareness and interoceptive accuracy: A review of the literature and directions for future research. Frontiers in Psychology, 5, 1379. doi:10.3389/fpsyg.2014.01379

Mariano, C. (2013). Holistic nursing: Scope and standards of practice. In B. Dossey \& L. Keegan (Eds.), Holistic nursing: A handbook for practice (6th ed., pp. 59-84). Burlington, MA: Jones \& Bartlett. 
Maslow, A. H. (1972). The farther reaches of human nature. New York, NY: Viking.

Mate, G. (2003). When the body says NO. New York, NY: John Wiley \& Sons.

McGorry, P. D., Chanen, A., McCarthy, E., van Riel, R., McKenzie, D., \& Singh, B. S. (1991). Posttraumatic stress disorder following recent-onset psychosis: An unrecognized postpsychotic syndrome. The Journal of Nervous and Mental Disease, 179, 253-258. doi:10.1097/00005053-199105000-00002

McKivergin, M. J. (1997). The nurse as an instrument of healing. In B. M. Dossey (Ed.), Core curriculum for holistic nursing (pp. 17-25). Gaithersburg, MD: Aspen.

McLaughlin, K. A., Koenen, K. C., Friedman, M. J., Ruscio, A. M., Karam, E. G., Shahly, V., . . Kessler, R. C. (2015). Subthreshold posttraumatic stress disorder in the World Health Organization world mental health surveys. Biological Psychiatry, 77(4), 375-384. doi:10.1016/j. biopsych.2014.03.028

McLean, C. P., \& Fitzgerald, H. (2016). Treating posttraumatic stress symptoms amoving people living with HIV: a criti cal review of intervention trials. Current Psychiatry Reports, 18(9), 83.

Mehta, S., \& Farina, A. (1997). Is being "sick" really better? Effect of the disease view of mental disorder on stigma. Journal of Social and Clinical Psychology, 16, 405-419. doi:10.1521/ jscp.1997.16.4.405

Meyer, H., Taiminen, T., Vuori, T., Aeijaelae, A., \& Helenius, H. (1999). Posttraumatic stress disorder symptoms related to psychosis and acute involuntary hospitalization in schizophrenic and delusional patients. Journal of Nervous and Mental Disease, 187, 343-352. doi:10.1097/00005053-199906000-00003

Miller-Karas, E. (2015). Building resilience to trauma. New York, NY: Routledge.

Mintzer, L., Stuber, M., Seacord, D., Castaneda, B. A., Mesrkhani, V., \& Glover, D. (2005). Traumatic stress symptoms in adolescent organ transplant recipients. Pediatrics, 115(6), 1640-1644. doi:10.1542/peds.2004-0118

Mol, S., Arntz, A., Metsemakers, J., Dinant, G.-J., Vilters-Van Montfort, P., \& Knottnerus, A. (2005). Symptoms of post-traumatic stress disorder after non-traumatic events: Events from an open population study. British Journal of Psychiatry, 186, 494-499. doi:10.1192/bjp.186.6.494

Moncher, F. J., \& Prinz, R. J. (1991). Treatment fidelity in outcome studies. Clinical Psychology Review, 11, 247-266. doi:10.1016/0272-7358(91)90103-2

Morrison, A., Frame, L., \& Larkin, W. (2003). Relationship between trauma and psychosis: A review and integration. British Journal of Clinical Psychology, 42, 331-353. doi:10.1348/014466503322528892

Morse, J. M., Solberg, S. M., Neander, W. L., Bottorff, J. L., \& Johnson, J. L. (1990). Concepts of caring and caring as a concept. Advances in Nursing Science, 13(1), 1-14.doi:10.1097/00012272-199009000-00002

Mota, N. P., Tsai, J., Sareen, J., Marx, B. P., Wisco, B. E., Harpaz-Rotem, L., . . Pietrzak, R. H. (2016). High burden of subthreshold DSM5 post-traumatic stress disorder in U.S. military veterans. World Psychiatry, 15 (2), 185-186. doi:10.1002/wps.20313

Najavits, L. M. (2002). Seeking safety: A treatment manual for PTSD and substance abuse. New York, NY: Guilford Press.

National Institute of Mental Health. (2009). Schizophrenia and bipolar disorder share genetic roots: Chromosomal hotspot of immunity/gene expression regulation implicated. Retrieved from https://www.nih.gov/news-events/news-releases/ schizophrenia-bipolar-disorder-share-genetic-roots

National Organization of Nurse Practitioner Faculties. (2013). Population-focused nurse practitioner competencies. Retrieved from https://c.ymcdn.com/sites/nonpf.site-ym.com/resource/ resmgr/competencies/populationfocusnpcomps2013.pdf

National Panel for Psychiatric Mental Health NP Competencies. (2003). Psychiatric-mental health nurse practitioner competencies. Washington, DC: National Organization of Nurse Practitioner Faculties. Retrieved from https://www.apna.org/files/public/NOPH_ COMPETENCIES.pdf

Nicholson, W., Durand, S., Vance, D., McGuinness, T., \& Carpenter, J. (2018). Trauma-based disorders and the cardio-neural mechanisms involved in dysfunctional self-regulation. Presented at the 2018 American Psychiatric Nurses Association Pre-Conference. Columbus, Ohio https:/ / e-learning.apna.org/products/1035-18-trauma-based-disorders-and-the-cardio-neuralmechanisms-involved-in-dysfunctional-self-regulation 
Norcross, J. C., \& Lambert, M. J. (2019). Psychotherapy relationships that work. (3rd ed., Vol. 1). New York, NY: Oxford University Press.

Ogden, P., Minton, K., \& Pain, C. (2006). Trauma and the body: A sensorimotor approach to psychotherapy. New York, NY: W. W. Norton.

Papale, L. A., Seltzer, L. J., Madrid, A., Pollak, S. D., \& Alisch, R. S. (2018). Differentially methylated genes in saliva are linked to childhood stress. Scientific Reports, 8, 10785. doi:10.1038/s41598-018-29107-0

Paradies, Y., Ben, J., Denson, N., Elias, A., Priest, N., Pieterse, A., Gupta, A., Kelaher, M., \& Gee, G. (2015). Racism as a determinant of health: A systematic review and meta-analysis. PloS one, 10(9), e0138511.

Parker, A. M., Sricharoenchai, T., Raparla, S., Schneck, K. W., Bienvenu, O. J., \& Needham, D. M. (2015). Post traumatic stress disorder in critical illness survivors: a metaanalysis. Critical Care Medicine, 43(5), 1121-1129.

Peplau, H. (1991). Interpersonal relations in nursing: A conceptual frame of reference for psychodynamic nursing. New York, NY: Springer Publishing Company.

Perry, B. D. (2001). The neurodevelopmental impact of violence in childhood. In D. Schetky \& E. P. Benedek (Eds.), Textbook of child and adolescent forensic psychiatry (pp. 221-238). Washington, DC: American Psychiatric Press.

Plakun, E., Sudak, D. M., \& Goldberg, D. (2009). The Y model: An integrated, evidence-based approach to teaching psychotherapy competencies. Journal of Psychiatric Practice, 15, 5-11. doi: $10.1037 / \mathrm{a} 0022123$

Porges, S. (2019, March 31). The emergence of polyvagal informed therapies in the treatment of trauma. Presented at The World Congress on Complex Trauma: Research | Intervention | Innovation.New York, NY.

Porges, S. W. (2011). The polyvagal theory. New York, NY: W. W. Norton.

Porges, S. W. \& Dana, D. (Eds.). (2018). Clinical applications of the polyvagal theory: The emergence of polyvagal informed therapies. New York, NY: W. W. Norton.

Raingruber, B. (2003). Nurture: The fundamental significance of relationship as a paradigm for mental health nursing. Perspectives in Psychiatric Care, 39(3), 104-112, 132-135. doi:10.1111/j.1744-6163.2003.00104.x

Read, J. (2010). Can poverty drive you mad? 'Schizophrenia', socio-economic status and the case for primary prevention. New Zealand Journal of Psychology, 39(2), 7-19. Retrieved from https://www.psychology.org.nz/wp-content/uploads/NZJP-Vol392-2010-2-Read.pdf

Robinson, J. S., \& Larson, C. (2010). Are traumatic events necessary to elicit symptoms of post-traumatic stress? Psychological Trauma: Theory, Research, Practice \& Policy, 2(2), 71-76. doi:10.1037/a0018954(2010)

Rogers, C. R. (1951). Client-centered therapy: Its current practice, implications, and theory. Boston, MA: Houghton Mifflin.

Sacks, V., \& Murphey, D. (2018, February 20). The prevalence of adverse childhood experiences, nationally, by state, and by race/ethnicity. Bethesda, MD: Child Trends. Retrieved from https: / www.childtrends.org/publications / prevalence-adverse-childhood-experiences-nationally-state-race-ethnicity

Safran, J. D., \& Muran, J. C. (2000). Negotiating the therapeutic alliance. New York, NY: Guilford Press.

SAMSHA (2020). Recovery and recovery support. Retrieved from https:/ / www.samhsa.gov/ find-help/recovery

Scaer, R. (2005). The trauma spectrum: Hidden wounds and human resiliency. New York, NY: W. W. Norton.

Schoenhofer, S. O. (2002). Philosophical underpinnings of an emergent methodology for nursing as caring inquiry. Nursing Science Quarterly, 15, 275-280. doi:10.1177/089431802320559173

Schore, A. (2019). Right brain psychotherapy. New York, NY: W. W. Norton.

Scott, J. (1979). Critical periods in organizational processes. In F. Falker \& J. Tanner (Eds.), Human growth neurobiology and nutrition (Vol. 3, pp. 223-243). New York, NY: Plenum Press.

Seng, J. S., Graham-Bermann, S. A., Clark, M. K., McCarthy, A. M., \& Ronis, D. L. (2005). Posttraumatic stress disorder and physical comorbidity among female children and adolescents: Results from service-use data. Pediatrics, 116(6), e767-e776. doi:10.1542/ peds.2005-0608 
Shapiro, F. (2012). Getting past your past: Take control of your life with self-help techniques from EMDR therapy. New York, NY: Rodale.

Shapiro, F. (2018). Eye movement desensitization and reprocessing (EMDR) (3rd ed.). New York, NY: Guilford Press.

Shaw, K., McFarlane, A., \& Bookless, C. (1997). The phenomenology of traumatic reactions to psychotic illness. The Journal of Nervous and Mental Disease, 185, 434-441. doi:10.1097/00005053-199707000-00003

Shonkoff, J. P. (2010). Building a new bio-developmental framework to guide the future of early childhood policy. Child Development, 81(1), 357-367. doi:10.1111/j.1467-8624.2009.01399.x

Shonkoff, J. P., \& Garner, A. S. (2012). The lifelong effects of early childhood adversity and toxic stress. Pediatrics, 129(1), 232-246. doi:10.1542/peds.2011-2663

Sibrava, N. J., Bjornsson, A. S., Perez Benitez, A. C. I., Moitra, E., Weisberg, R. B., \& Keller, M. B. (2019). Posttraumatic stress disorder in African American and Latinx adults: Clinical course and the role of racial and ethnic discrimination. American Psychologist, 74(1), 101-116.

Siegel, D. (2012). The developing mind, 2nd edition. New York, NY: The Guilford Press.

Siegel, R. S., \& Rosen, L. C. (1962). Character style and anxiety tolerance: A study of intrapsychic change. In H. Strupp \& L. Luborsky (Eds.), Research in psychotherapy (Vol. 2, pp. 206-217). Washington, DC: American Psychological Association.

Siu, B. W., Ng, B. F., Li, V. C., Yeung, Y.-M., Lee, M. K., \& Leung, A. Y. (2012). Mental health recovery for psychiatric inpatient services: Perceived importance of the elements of recovery. East Asian Archive of Psychiatry, 22, 39-48. Retrieved from https:/ /www.easap.asia/index. $\mathrm{php} /$ find-issues/past-issue/item/151-1202-v22n2-39-oa

Smoller, J. W. (2016). The genetics of stress-related disorders: PTSD, depression, and anxiety disorders. Neuropsychopharmacology, 14(1), 297-319. doi:10.1038/npp.2015.266

Smoyak, S. (1990). The nurse psychotherapist as unique practitioner. In J. Durham \& S. Hardin (Eds.), The nurse psychotherapist in private practice (pp. 15-24). New York, NY: Springer Publishing Company.

Souza-Talarico, J. N., Plusquellec, P., Lupien, S. J., Fiocco, A., \& Suchecki, D. (2014). Crosscountry differences in basal and stress-induced cortisol secretion in older adults. PLOS ONE, 9(8), e105968. doi:10.1371/journal.pone.0105968

Steele, A. (2007). Developing a secure self: An attachment-based approach to adult psychotherapy. Gabriola, BC, Canada: Author.

Stein, L. A., Goldmann, E., Zamzam, A., Luciano, J. M., Messe, S. R., Cucchiara, B. L., Kasner, S. E., \& Mullen, M. T. (2018). Association between anxiety, depression, and post-traumatic stress disorder and outcomes after ischemic stroke. Frontiers in Neurology, 9, 890.

Stien, P., \& Kendall, J. (2006). Psychological trauma and the developing brain. New York, NY: Hawthorne Press.

Strupp, H. H., \& Anderson, T. (1997). On the limitations of treatment manual. Clinical Psychology: Science and Practice, 4, 76-82. doi:10.1111/j.1468-2850.1997.tb00101.x

Substance Abuse and Mental Health Services Administration [SAMHSA]. (2012) Trauma and justice strategic initiative. Retrieved from https:/ / www.ncbi.nlm.nih.gov/books/ NBK207192/

Substance Abuse and Mental Health Services Administration. (2012a). With peer support, recovery is possible. SAMHSA Newsletter, 20(3), 6-7. Retrieved from https:/ / taadas. s3.amazonaws.com/files/0fd5cc121bdcc1b4089d24665849b552-Preventing\%20Suicide\%20 Across $\% 20$ the $\% 20$ Nation $\% 20 F a l l \% 202012$.pdf

Substance Abuse and Mental Health Services Administration. (2020). Recovery https://www. samhsa.gov/find-help/recovery. Retrieved from http://www.apna.org/files/public/Recovery_ to_Practice_Overview.pdf

Suglia, S. F., Koenen, K. C., Boynton-Jarrett, R., Chan, P. S., Clark, C. J., Danese, A., . . . Zachariah, J. P. (2018). Childhood and adolescent adversity and cardiometabolic outcomes: A scientific statement from the American Heart Association. Circulation, 137(5), e15-e28. doi:10.1161/ CIR.0000000000000536

Substance Abuse and Mental Health Services Administration. (n.d) Resilience \& Stress Management Retrieved from https://www.samhsa.gov/dbhis-collections/ resilience-stress-management 
Sullivan, H. S. (1947). Conceptions of modern psychiatry. Washington, DC: William Alanson White Institute.

Teicher, M., Polcari, A., Andersen, S., Anderson, C. M., \& Navalta, C. (2003). Neurobiological effects of childhood stress and trauma. In S. Coates, J. Rosenthal, \& D. Schechter (Eds.), September 11: Trauma and human bonds (pp. 211-238). Hillsdale, NJ: Analytic Press.

Thomas, J. D., Finch, L. P., Schoenhofer, S. O., \& Green, A. (2004). The caring relationships created by nurse practitioners and the ones nursed: Implications for practice. Topics in Advanced Practice Nursing eJournal, 4(4). Retrieved from https://www.medscape.com/viewarticle/496420

Truijens, F., Zühlke van Hulzen, L., \& Vanheule, S. (2019). To manualize, or not to manualize: Is that still the question? A systematic review of empirical evidence for manual superiority in psychological treatment. Journal of Clinical Psychology, 75(3), 329-343. doi:10.1002/jclp.22712

Tryon, G. S., \& Winograd, G. (2002). Goal consensus and collaboration. In J. Norcross (Ed.), Psychotherapy relationships that work (pp. 109-125). New York, NY: Oxford University Press.

van Dam, D. S., van der Ven, E., Velthorst, E., Selten, J. P., Morgan, C., \& de Haan L. (2012). Childhood bullying and the association with psychosis in non-clinical and clinical samples: A review and meta-analysis. Psychological Medicine, 42(12), 2463-2474. doi:10.1017/ S0033291712000360

Vanderhoef, D. M., \& Delaney, K. R. (2017). National organization of nurse practitioner faculties: Psychiatric mental health survey. Journal of the American Psychiatric Nurses Association, 23, 159-165. doi:10.1177/1078390316685154

van der Kolk, B. (2014). The body keeps the score. New York, NY: Penguin Books.

Varese, F., Smeets, F., Drukker, M., Lieverse, R., Lataster, T., Viechtbauer, W., . . Bentall, R. P. (2012). Childhood adversities increase the risk of psychosis: A meta-analysis of patientcontrol, prospective and cross-sectional cohort studies. Schizophrenia Bulletin, 38(4), 661-671. doi:10.1093/schbul/sbs050

Wachtel, P. (2011). Therapeutic communication: Knowing what to say when (2nd ed.). New York, NY: Guilford Press.

Watson, J. (2012). Jean Watson's theory of caring. Retrieved from http:/ / currentnursing.com/ nursing_theory/Watson.html

Wheeler, K. (2011). A relationship-based model for psychiatric nursing practice. Perspectives in Psychiatric Care, 47(3), 151-159. doi:10.1111/j.1744-6163.2010.00285

Wheeler, K., \& Delaney, K. (2005). Challenges and realities of teaching psychotherapy: A survey of psychiatric-mental health nursing graduate programs. Perspectives in Psychiatric Care, 44(2), 72-80. doi:10.1111/j.1744-6163.2008.00156.x

Wheeler, K., \& Haber, J. (2004). Development of psychiatric nurse practitioner competencies: Opportunities for the 21st century. Journal of the American Psychiatric Nursing Association, 10(3), 129-138. doi:10.1177/1078390304266218

Wheeler, K. \& Phillips, K. (2019). The development of trauma and resilience competencies for nursing education. Journal of the American Psychiatric Nurses Association. Advance online publication. doi:10.1177/1078390319878779

Williams, M. T., Metzger, I. W., Leins, C., \& DeLapp, C. (2018). Assessing racial trauma within a DSM-5 frame work: The UCONN Racial/Ethnic Stress \& Trauma Survey. Practice Innovations, $3(4), 242-260$.

World Health Organization. (2016). Child maltreatment: Key facts. Retrieved from http:/ /www. who.int/news-room/fact-sheets/detail/child-maltreatment

Yalom, I. D., \& Ferguson, N. (2002). The gift of therapy: An open letter to a new generation of therapists and their patients. New York, NY: Harper Collins. 


\section{APPENDIX 1.1 \\ Suggestions for Presenting a Case}

Presenting a case can seem overwhelming, especially with complex patients. The following guidelines are intended to help you organize your thinking, summarize salient information about your patient in a coherent manner, identify areas where the therapy is stuck (resistance), and formulate questions that may offer insight into the process. Identifying information should be disguised.

\section{BASIC INFORMATION}

Demographics: age, race/ethnicity, gender, sexual orientation, education, and occupation.

Family: relationship status, living arrangement, members of immediate family, and extended relevant family members.

Working Diagnosis and Symptoms: dissociation, anxiety, depression, eating disorder, substance abuse, self-injury, and suicide attempts, destructive or violent behavior.

Relevant Medical Problems and Physical Disabilities: diabetes, asthma, chronic pain, birth defects, sensory impairment, impaired mobility, and so on.

Patient's Coping Mechanisms: both healthy and unhealthy, defenses, and ego functioning.

Treatment History: inpatient, outpatient, how long and intensive, treatment failures and responses.

Current Treatment: inpatient, outpatient, partial individual, group, and family.

Medication(s): current and past history.

\section{CASE CONCEPTUALIZATION}

1. What are the reasons the patient came for treatment now?

2. What are the patient's goals? How would the person know if the treatment was successful?
3. When did the current symptoms start?

4. What other situations may be contributing to the problem now?

5. Speculate on what experiential contributors from the past might be driving the current symptoms?

6. Is there a current crisis?

7. Resources and strengths.

8. Draw a timeline with the patient of the most disturbing and pleasant events in the person's life and rate disturbances on a 0 to 10 scale with 10 being the most disturbing. See Chapter 13 for example of timeline.

\section{QUESTIONS TO PONDER}

What's going well in the therapeutic process, and what is problematic? Have you established a therapeutic alliance? Is the patient's life stabilized? Is the patient avoiding or working on issues? Undermining the therapy? Flooding with memories or decompensating?

What makes you want to present this patient? What's unusual, special, difficult, confusing, arousing, frustrating, scary, overwhelming?

What do you experience with this patient that is unusual for you? Do you feel intense emotions, like or dislike, anger, admiration, humiliation, fear, revulsion, sleepy, dizzy, disoriented, a desire to nurture or rescue, and the urge to confront. Do you wish you could get rid of this patient, or are you afraid of losing him or her?

\section{TREATMENT HIERARCHY}

Based on this information and the hierarchy of treatment in your book, what do you think is the most appropriate interventions/treatment for this person now? What are treatment priorities? 


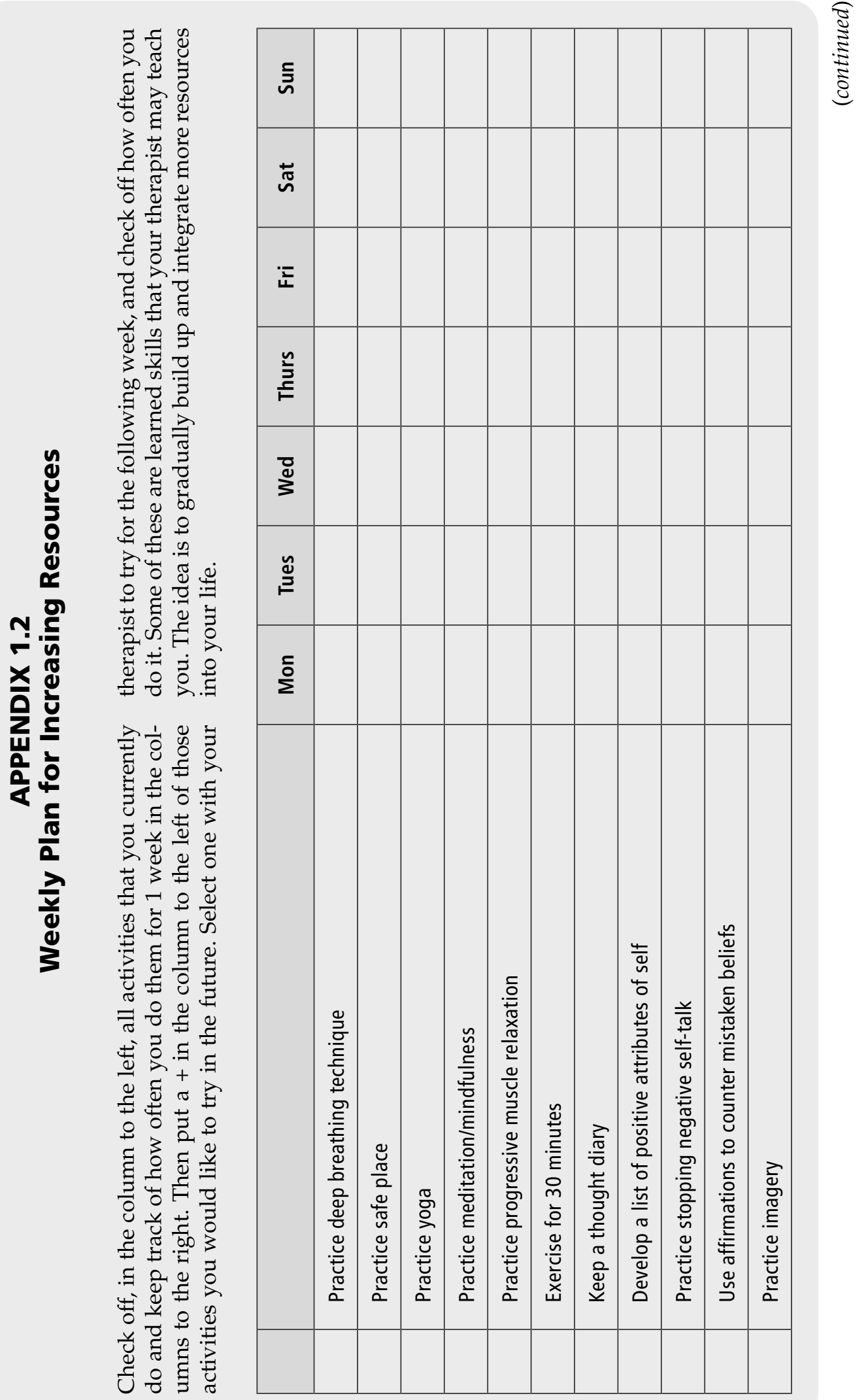




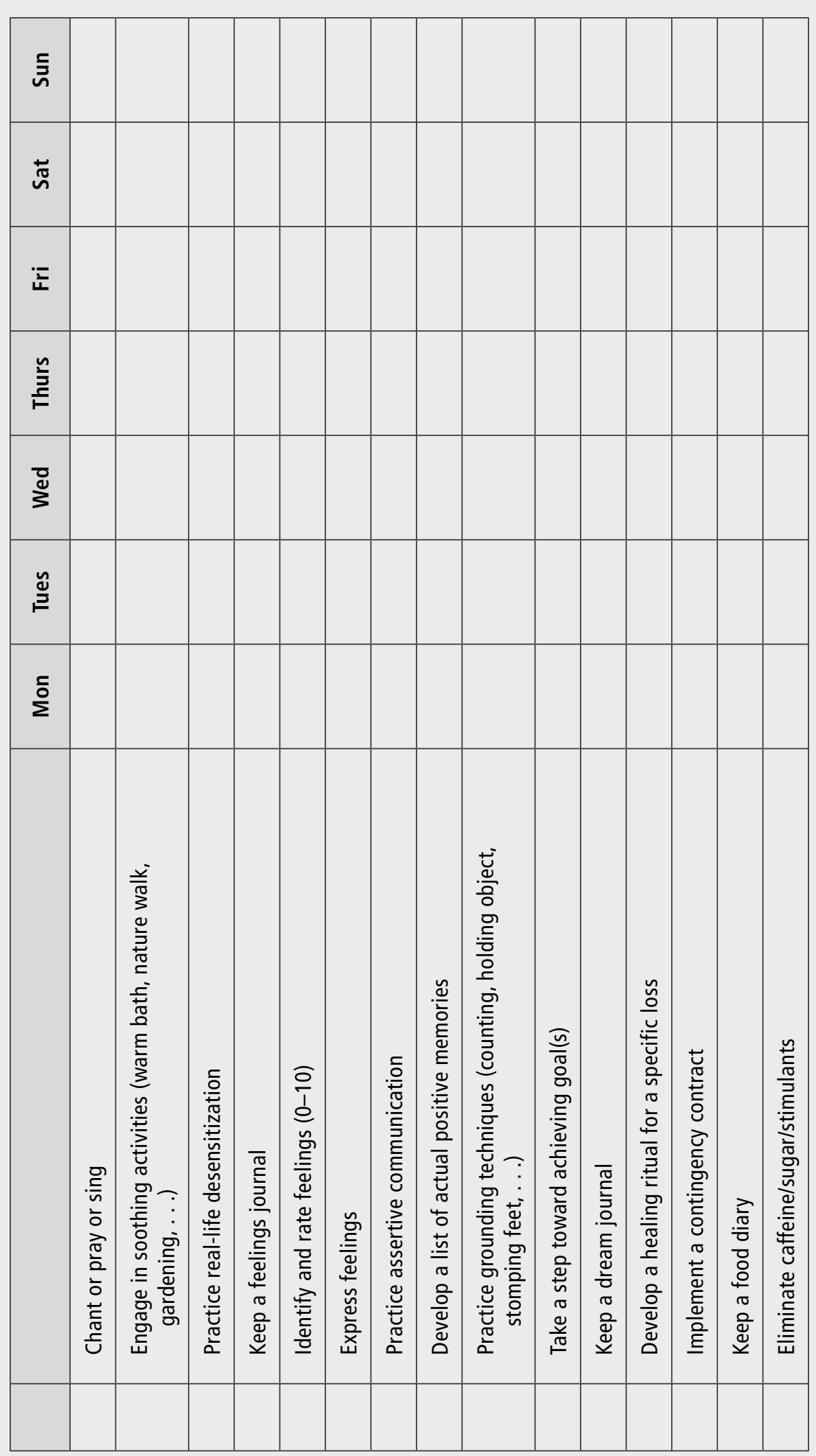




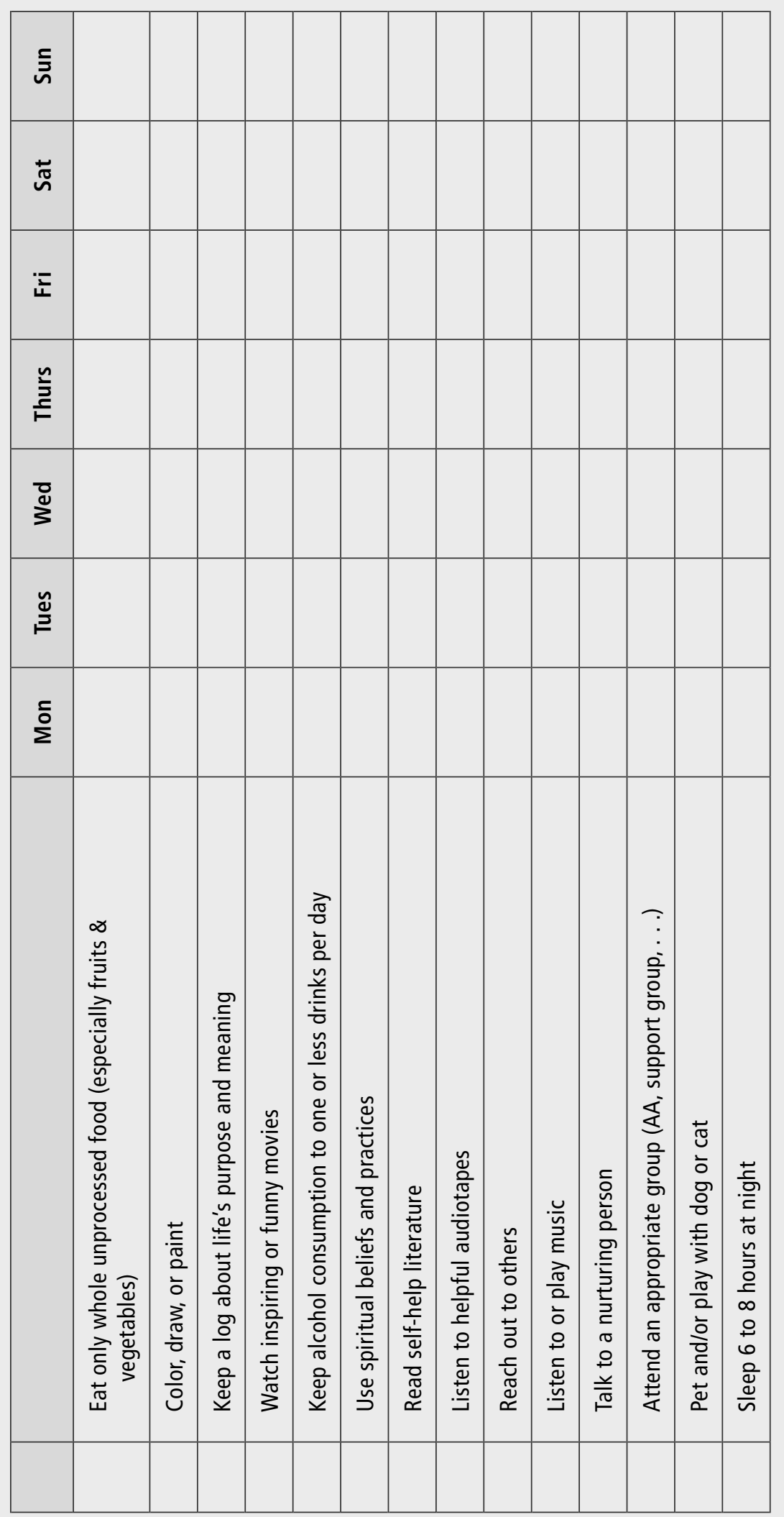




\section{APPENDIX 1.3}

Weekly Plan

Please fill in two to three goals for the week and check off each day that you meet that goal.

\begin{tabular}{|c|c|c|c|c|c|c|c|}
\hline Goal & Mon & Tues & Wed & Thurs & Fri & Sat & Sun \\
\hline & & & & & & & \\
\hline & & & & & & & \\
\hline & & & & & & & \\
\hline
\end{tabular}




\section{APPENDIX 1.4 \\ Treatment and Case Management}

Patient:

Address:

Date:

Phone:

Insurance:

Note: At the end of this form is the form for Patient Case Management Needs, which patients can fill out before the session to identify their key areas of need. However, it is still important for the therapist to assess each goal directly, because patients may not be aware of some needs.

\section{Housing Characteristics}

Goal Stable and safe living situation.

Notes Unhealthy living situations include short-term shelter, living with a person who abuses substances, an unsafe neighborhood, and a domestic violence situation.

Status If the goal is already met, check here and describe. If the goal is not met, check here and fill out the Case Management Goal Sheet.

\section{Individual Psychotherapy}

Goal Treatment that patient finds helpful.

Notes Try to get every patient into individual psychotherapy. Inquire whether the patient has any preferences (e.g., gender, theoretical orientation).

Status If the goal is already met, check here and describe.

If the goal is not met, check here and fill out the Case Management Goal Sheet.

\section{Psychiatric Medication}

Goal Treatment that patient finds helpful for psychiatric symptoms (e.g., depression, sleep problems) or substance abuse (e.g., naltrexone for alcohol cravings).

Notes If the patient has never had a psychopharmacologic evaluation, one is strongly recommended, unless the patient has serious objections; even then, evaluation and information are helpful before making a decision.

Status If the goal is already met, check here If the goal is not met, check here and describe. ment Goal Sheet.

\section{HIV Testing and Counseling}

Goal Test as soon as possible, unless one was completed in the past 6 months and there have been no high-risk behaviors since then. For a patient at risk for HIV infection who is unwilling to get testing and counseling, it is strongly suggested that the therapist hold an individual session with the patient to explore and encourage these goals.

Notes Assist patient with accessing community resources in your geographic area.

Status If the goal is already met, check here and describe. 
If the goal is not met, check here and fill out the Case Management Goal Sheet.

\section{Job, Volunteer Work, and School}

Goal At least 10 hours per week of scheduled productive time.

Notes If the patient is unable to meet the goal of 10 hours/week, have the patient hand in a weekly schedule with constructive activities out of the house (e.g., library, gym).

Status If the goal is already met, check here and describe.

If the goal is not met, check here and fill out the Case Management Goal Sheet.

\section{Self-Help Groups and Group Therapy}

Goal As many groups as the patient is willing to attend.

Notes Elicit the patient's preferences, and consider a wide range of options (e.g., dual-diagnosis groups, women's groups, veterans' groups). For self-help groups (e.g., Alcoholics Anonymous), give the patient a list of local groups, strongly encourage attendance, and mention that the sessions are free. However, do not insist on self-help groups or convey negative judgment if the patient does not want to attend. If the patient participates in self-help groups, encourage seeking a sponsor.

Status If the goal is already met, check here and describe.

If the goal is not met, check here and fill out the Case Management Goal Sheet.

\section{Day Treatment}

Goal As needed and based on the patient's level of impairment, ability to attend a day program, and schedule.

Notes If possible, locate a specialty day program (e.g., substance abuse, PTSD). If the patient is able to attend (e.g., job, school, volunteer activity), do not refer to day treatment, because it is usually better to have the patient keep working; however, if the patient is working part-time, some programs allow partial attendance.

Status If the goal is already met, check here If the goal is not met, check here and describe. ment Goal Sheet.

\section{Detoxification and Inpatient Care}

Goal To obtain an appropriate level of care.

Notes Detox is necessary if the patient's use is so severe that it represents a serious danger (e.g., likelihood of suicide, causing severe health problems, withdrawal requires medical supervision, such as for painkillers or severe daily alcohol use). If the patient is not in acute danger but cannot get off substances, detox may or may not be helpful; many patients are able to stay off substances during the detox but return to their usual living environment and go back to substance use. For such patients, helping set up adequate outpatient supports is usually preferable. Inquiring about patient's history (e.g., number of past detox episodes and their impact) can be helpful in making a decision.

Psychiatric inpatient care is typically recommended if the patient is a serious suicide or homicide risk ${ }^{*}$ (i.e., not simply ideation, but immediate plan, intent, and inability to contract for safety) or the patient's psychiatric symptoms are so severe that functioning is impaired (e.g., 
psychotic symptoms prevent a mother from caring for her child). In some circumstances, the patient may need to be involuntarily committed; seek supervision and legal advice on this topic.

Status If the goal is already met, check here and describe. If the goal is not met, check here and fill out the Case Management Goal Sheet.

\section{Parenting Skills and Resources for Children}

Goal If the patient has children, inquire about parenting skills training and about referrals to help the children obtain treatment, health insurance, and other needs.

Notes You may need to gently inquire to assess whether the patient's children are being abused or neglected. If so, you are required by law to report it to your local protective service agency. The same rule applies for elder abuse or neglect.

Status If the goal is already met, check here and describe. If the goal is not met, check here and fill out the Case Manage-

10. Medical Care ment Goal Sheet.

Goals Annual examinations for (a) general health, (b) vision, (c) dentistry, and (d) gynecology (for women), including (e) instruction about adequate birth control and prevention of sexually transmitted diseases.

Notes Other medical care may be needed if the patient has a particular illness.

Status If all five goals are already met, check here and describe.

If any of the five goals is not met or other medical issues need attention, check here and fill out the Case Management Goal Sheet for each.

11. Financial Assistance (e.g., food stamps, Medicaid)

Goal Health insurance and adequate finances for daily needs.

Notes It is crucial to help the patient obtain health insurance and entitlement benefits (e.g., food stamps, Medicaid), if needed. The patient may need help filling out the forms; the patient may be unable to manage the task alone, because the bureaucracy of these programs can be overwhelming. If much help is needed, you may want to refer the patient to a social worker or other professional skilled in this area. If the patient is a parent, be sure to check whether the children are eligible.

Status If the goal is already met, check here If the goal is not met, check here and describe. ment Goal Sheet.

\section{Leisure Time}

Goal At least 2 hours per day in safe leisure activities.

Notes Leisure includes socializing with safe people and activities such as hobbies, sports, outings, and movies. Some patients are so overwhelmed with responsibility that they do not find time for themselves. Adequate leisure is necessary for maintaining a healthy lifestyle.

Status If the goal is already met, check here and describe.

If the goal is not met, check here and fill out the Case Management Goal Sheet.

\section{Domestic Violence and Abusive Relationships}

Goal Freedom from domestic violence and abusive relationships.

Notes It may be extremely difficult to get the patient to leave a situation of domestic violence. Be sure to consult a supervisor and a domestic violence hotline representative. 
Status If the goal is already met, check here and describe.

If the goal is not met, check here ment Goal Sheet.

14. Impulses to Harm Self or Others (e.g., suicide, homicide)

Goal Absence of such impulses, or if such impulses are present, a clear and specific safety plan is in place.

Notes Many patients have thoughts of harming self or others; however, to determine whether the patient is at serious risk for action and how to manage this risk, see the guidelines developed by the International Society of Study for Dissociative Disorders in Chapter 3.

Status If the goal is already met, check here and describe.

If the goal is not met, check here and fill out the Case Management Goal Sheet.

15. Alternative Treatments (e.g., acupuncture, meditation)

Goal The patient is informed about alternative treatments that may be beneficial.

Notes Patients should be informed that some people in early recovery benefit from acupuncture, meditation, and other nonstandard treatments. Try to identify local referrals for such resources.

Status If the goal is already met, check here and describe.

If the goal is not met, check here and fill out the Case Management Goal Sheet.

16. Self-Help Books and Materials

Goal The patient is offered one or two suggestions for self-help books and other materials, such as audiotapes or Internet sites, that offer education and support.

Notes All patients should be encouraged to use self-help materials outside of sessions as much as possible. For patients who do not like to read, alternative modes (e.g., audiotapes) are suggested. Self-help can address PTSD, substance abuse, or any other life problems (e.g., study skills, parenting skills, relationship skills, leisure activities, and medical problems).

Status If the goal is already met, check here and describe.

If the goal is not met, check here and fill out the Case Manage-

\section{Additional Goal} ment Goal Sheet.

Goal

Notes

*For homicide risk or any other intent to physically harm another person, the therapist must follow "duty to warn" legal standards, which usually involve an immediate warning to the specific person the patient plans to assault. Always seek supervision and legal advice, and be knowledgeable in advance about how to manage such a situation.

\section{CASE MANAGEMENT GOAL SHEET}

Patient:

Date:

Goal: 
Referrals given to patient, date given, and deadline (if any) for each:

Describe patient's motivation to work on this goal:

Emotional obstacles that may hinder completion (and strategies implemented to help patient overcome these):

Therapist to do:

Follow-up (date and update):

\section{PATIENT CASE MANAGEMENT NEEDS}

\section{Do you need help with any of the following? (circle one)}

\begin{tabular}{|l|l}
\hline 1. Housing characteristics & Yes/Maybe/No \\
\hline 2. Individual psychotherapy & Yes/Maybe/No \\
\hline 3. Psychiatric medication & Yes/Maybe/No \\
\hline 4. HIV testing and counseling & Yes/Maybe/No \\
\hline 5. Job, volunteer work, and school & Yes/Maybe/No \\
\hline 6. Self-help groups and group therapy & Yes/Maybe/No \\
\hline 7. Day treatment & Yes/Maybe/No \\
\hline 8. Detoxification and inpatient care & Yes/Maybe/No \\
\hline 9. Parenting skills and resources for children & Yes/Maybe/No \\
\hline 10. Medical care & Yes/Maybe/No \\
\hline 11. Financial assistance (e.g., food stamps, Medicaid) & Yes/Maybe/No \\
\hline 12. Leisure time & Yes/Maybe/No \\
\hline 13. Domestic violence and abusive relationships & Yes/Maybe/No \\
\hline 14. Impulses to harm self or others (e.g., suicide, & Yes/Maybe/No \\
\hline homicide) & \\
\hline 15. Alternative treatments (e.g., acupuncture, meditation) & Yes/Maybe/No \\
\hline 16. Self-help books and materials & Yes/Maybe/No \\
\hline 17. Additional goal & Yes/Maybe/No \\
\hline
\end{tabular}

Permission to photocopy this form is granted to purchasers of this book for personal use only. Source: Adapted from Najavits, L. M. (2002). Seeking safety: A treatment manual for PTSD and substance abuse. New York, NY: Guilford Press. 


\section{APPENDIX 1.5 \\ Stage I}

\section{STABILIZATION CHECKLIST}

Please check all indicators below to help assess whether patient is stabilized and ready to move to Stage II.

\begin{tabular}{|l|l|}
\hline & Comfort with own body and physical experience \\
\hline Patient is able to establish a useful distance from the traumatic event \\
\hline No current life crisis such as impending litigation or medical problems \\
\hline Patient accepts diagnosis and has a working knowledge of trauma \\
\hline Patient's mood is stable, even if depressed \\
\hline Patient has at least two or more people to count on \\
\hline Patient knows and uses self-soothing techniques \\
\hline Patient gives honest self-reports \\
\hline Patient's living situation is stable \\
\hline Patient is able to communicate \\
\hline Patient has stable therapeutic relationship and adequate trust of others \\
\hline Patient has adequate impulse control, no injurious behavior to self or others \\
\hline \begin{tabular}{l} 
Patient stays grounded and oriented x3 when distressed \\
\hline No major dissociation present
\end{tabular} \\
\hline Patient can identify triggers and reports significant symptoms \\
\hline Patient can set limits and is able to leave dangerous situations if necessary \\
\hline Patient can tolerate positive and negative affect, and shame \\
\hline
\end{tabular}




\section{APPENDIX 1.6 \\ Stage II}

\section{PROCESSING CHECKLIST}

Please check all indicators below to help assess whether patient has adequately processed trauma and is moving to Stage III, future visioning. The stabilization checklist should already have been achieved.

\begin{tabular}{|l|l|}
\hline & No significant affect changes \\
\hline & Self-referencing cognitions are positive in relation to past event \\
& Can dismiss thoughts of trauma at will \\
\hline & Relationships are adaptive \\
\hline Work is productive \\
\hline & \begin{tabular}{l} 
Good quality of decision-making \\
\hline
\end{tabular} \\
\hline Creativity begins to emerge \\
\hline Complaints tend to deal with present-day events \\
\hline Affect is proportionate to current events \\
\hline Congruence between behavior, thoughts, and affect \\
\hline
\end{tabular}




\section{APPENDIX 1.7 Safe-Place Exercise}

The safe-place exercise described below helps the patient to enhance skills during stabilization as well as to decrease distress after processing. Through the ability to create one's own safe place, the person is empowered. As with all learning, the more it is practiced, the more readily available it is when needed. Thus, it should be used on a day-to-day basis. If a patient feels there is no place - real or imaginary - that is safe, have the patient focus on one time in his or her life when he or she felt safe or on a person he or she admires who exemplifies positive attributes, such as strength or control. If the person still cannot find a safe place, ask the person to think of a place where he or she feels relaxed or comfortable. Sometimes patients become more distressed when they relax and it may take some time before the person is able to identify a positive resource. Identifying a safe place resource may take several sessions. Ask the person to sit with his or her feet firmly planted on the floor. Sometimes this exercise is conducted with soothing music and/or background nature sounds. Some therapists tape the exercise with their voice to give to the patient to practice at home. The safe-place exercise follows.

Ask the person to identify an image of a safe place that he or she can easily evoke that creates a personal feeling of calm and safety. Use soothing tones to enhance the imagery, asking the person to "see what you see," "feel what you feel," "notice the sounds, smells, and colors in your special place." Once identified, ask the person to focus on the image, feel the emotions, and identify the location of the pleasing physical sensations and where he or she is in the body. "Concentrate on those pleasant sensations in your body and just enjoy as you breathe deeply, relaxing and feeling safe." After you have slowly deepened his or her experience of this, slowly ask the person to come back and tell you a description of the place. Ask for details so that you can assist the person in accessing this place in the future. Ask how he or she feels and if the experience has been difficult for the person and/or no positive emotions are experienced, explore other resources that might be helpful. If at any time the person indicates that he or she is not feeling safe, the exercise should be stopped immediately.

If successful in accessing a safe place, the person is asked for a single word that fits the picture (i.e., beach, forest ...) and then asked to repeat the exercise using the person's words for the experience along with deep breathing. Then ask the person to repeat on his or her own, bringing up the image, emotions, and body sensations. Reinforce, after this exercise, that his or her safe place can be used as a resource and ask the patient to practice over the next week, once a day.

During the next session, practice again with the person. Then ask the patient to bring up a minor annoyance and notice the negative feelings while guiding the person through the safe place until the negative feelings have dissipated. Then ask the person to bring up a negative disturbing thought once again and to access the safe place but this time on his or her own without your assistance.

Occasionally the safe-place exercise triggers intense negative affect. Patients should be made aware about the possible activation of issues during the safeplace exercise. Reassure the person that even if temporary activation of issues does occur, this is not beyond the limits of expectation, and that it may identify issues that will be addressed in the course of therapy anyway. 


\section{APPENDIX 1.8 Container Exercise}

This exercise is an important affect management strategy that can be taught to the patient and practiced so that the person can feel in control and develop mastery over his or her emotions. It also assists with self-soothing, decreasing arousal, and reinforcing a sense of safety. The person should already have a safe place. This exercise should be initiated toward the end of the session when the person has intense negative feelings of anxiety, anger, fear, and/or sadness.

The therapist introduces by saying something like: "Did you know that we can put those bad feelings into a container so you won't feel so overwhelmed when you leave?" The person's curiosity is usually piqued at this point even if he or she does not believe you. Continue with: "I can help you do this and then you can take out those feelings when you want and deal with them the next time we meet or when you decide it is okay." Usually the person agrees if for no other reason than he or she is curious and may think you are really strange to suggest such a thing. The therapist continues in a soothing tone: "So, just imagine you have a container; you can close your eyes or not as you wish. It can be made out of anything that you want and be any size you want but be sure it has a tight lid that you can cover or lock because we are going to put all those negative feelings in. Let me know once you have an image in your head." Once the person says he or she has the image, ask him or her for a few details regarding size and so on. Then ask the patient to "return to the image and imagine all those bad feelings going into the container. Once you have all the bad feelings in the container, lock it up. Let me know when they are in there." Once the person says they are in the container, ask the person whether there is any percentage that is still not in the container and usually the person will say something like $10 \%$ or $20 \%$. At that point, ask the person: "Do you need a bigger container to accommodate all the bad feelings? You can make it as big as you want. See whether you can put the rest of those feelings in the container now. Let me know when the rest of the feelings are all in the container and locked." If more negative feelings come up, continue with either imaging another container or making the one he or she has bigger. Ask the person what this was like for him or her, checking to see whether he or she is okay.

It is important to do this exercise slowly and use pacing so that the person does not feel rushed. The session can then be ended with the safe-place exercise. Ask the person to practice the container exercise during the week when negative feelings come up. The patient can also practice allowing the feelings to come out if he or she thinks he or she can manage this and journal about these feelings between sessions. Asking the person at the next session: "What was different for you this past week?" and exploring how feelings were or were not manageable are important follow-up steps and help to assess how to increase the effectiveness of this exercise. 